Cavendish-HEP-02/04

DESY 02-114

hep-ph/0208130

\title{
NLO Corrections to the Photon Impact Factor: Combining Real and Virtual Corrections
}

\author{
J. Bartels $^{(a) *}$, D. Colferai ${ }^{(a) * \dagger}$, S. Gieseke ${ }^{(b) *}$, A. Kyrieleis ${ }^{(a) * \ddagger}$ \\ (a) II. Institut für Theoretische Physik, Universität Hamburg, \\ Luruper Chaussee 149, 22761 Hamburg, Germany \\ (b) Cavendish Laboratory, University of Cambridge, \\ Madingley Road, Cambridge CB3 OHE, U.K.
}

\begin{abstract}
In this third part of our calculation of the QCD NLO corrections to the photon impact factor we combine our previous results for the real corrections with the singular pieces of the virtual corrections and present finite analytic expressions for the quark-antiquark-gluon intermediate state inside the photon impact factor. We begin with a list of the infrared singular pieces of the virtual correction, obtained in the first step of our program. We then list the complete results for the real corrections (longitudinal and transverse photon polarization). In the next step we define, for the real corrections, the collinear and soft singular regions and calculate their contributions to the impact factor. We then subtract the contribution due to the central region. Finally, we combine the real corrections with the singular pieces of the virtual corrections and obtain our finite results.
\end{abstract}

\section{INTRODUCTION}

This paper represents the third part of our investigation of the photon impact factor in next-to-leading order QCD. First results of the calculation of NLO corrections to the photon impact factor have been published in [1] and [2]. In [四] we have computed the QCD NLO corrections to the process $\gamma^{*}+q \rightarrow(q \bar{q})+q$ which lead to the virtual corrections of the photon impact factor. The second paper, [2], contains the process $\gamma^{*}+q \rightarrow(q \bar{q} g)+q$, leading to

\footnotetext{
*supported by the EU TMR-Network 'QCD and the Deep Structure of Elementary Particles', contract number FMRX-CT98-0194 (DG 12-MIHT).

${ }^{\dagger}$ Alexander von Humboldt Fellow.

${ }^{\ddagger}$ supported by the Graduiertenkolleg 'Zukünftige Entwicklungen der Teilchenphysik'.
} 
the real corrections of the photon impact factor. Results are given for the helicity-summed squared matrix elements, with the longitudinally polarized photon in the initial state. In the present paper we will complete this part of our calculation by listing also the results for the transverse polarization of the photon.

The main purpose of the present paper is the combination of singular pieces of virtual and real corrections. To begin with the former ones, in [1] we have listed the results for the virtual corrections which contain both the finite and the singular pieces. As the first step we therefore have to separate the sum of all singular $1 / \epsilon$ terms from the finite part. In the next step we turn to the real corrections. Infrared singularities will come from those kinematic regions of the $q \bar{q} g$ system where the gluon is either soft or collinear with the quark or with the antiquark. We compute the soft and the collinear approximations of our matrix elements, and, by subtracting these divergent contributions from the full matrix elements, we form finite combinations. Finally, by combining the singular pieces of the real corrections with those coming from the virtual corrections, we obtain further finite terms which have the form of the Born approximation and can be computed analytically. As the main result of this paper, we present finite expressions for the $q \bar{q} g$ contributions to the photon impact factor. As the fourth and remaining part of our program we will be left with the task of evaluating numerically the finite integrals obtained in this paper, as well as the finite pieces from the virtual corrections.

This paper will be organized as follows. After the definition of the impact factor (section III) we first (section III) list the infrared singular pieces of the virtual corrections. We then turn to the real corrections and present the complete results for the real corrections, both for the longitudinal and for the transverse photon (section IVD). In the following section $\mathrm{Q}$ we compute the collinear and the soft limits of the real corrections. We discuss in detail the definition of the impact factor correction in section $\nabla \mathbb{1}$, where the subtraction of the central region, i.e. the leading $\log s$ term, is discussed. In section VII we complete our program, by combining the singular pieces of the real corrections with those of the virtual corrections and by demonstrating their infrared finiteness. In a final section we summarize our results and give a brief outline of the remaining part of our program.

\section{DEFINITION OF THE IMPACT FACTOR}

We consider the elastic scattering of two particles $A$ and $B$ with momenta $p_{A}$ and $p_{B}$ in the Regge limit $s \rightarrow \infty$, where the momentum transfer $t=q^{2}$ is kept fixed. In leading order in $\alpha_{s}$ we write the scattering amplitude $T_{A B}$ in the form:

$$
T_{A B}^{(0)}\left(s, t=q^{2}\right)=i s \int \frac{d^{D-2} \boldsymbol{r}}{(2 \pi)^{D-2}} \tilde{\Phi}_{A}^{(0)} \frac{1}{\boldsymbol{r}^{2}} \frac{1}{(\boldsymbol{q}-\boldsymbol{r})^{2}} \tilde{\Phi}_{B}^{(0)} .
$$

The impact factors $\Phi_{A}^{(0)}$ and $\Phi_{B}^{(0)}$ for the external particles (quarks, gluons, or photons) are of order $\alpha_{s}$. The exchanged gluons carry transverse momenta $\boldsymbol{r}$ and $(\boldsymbol{q}-\boldsymbol{r})$ respectively. Taking the cut amplitude we define the total cross section for the scattering of $A$ and $B$ via the optical theorem as

$$
\sigma_{A B}^{(0)}=\frac{1}{s} \operatorname{Im} T_{A B}^{(0)}(s, t=0)=\int \frac{d^{D-2} \boldsymbol{r}}{(2 \pi)^{D-2}} \Phi_{A}^{(0)} \frac{1}{\boldsymbol{r}^{4}} \Phi_{B}^{(0)} .
$$


Here we have dropped the tilde symbol on the impact factors to denote the additional $s$ channel cut. This equation can be used as the definition of the leading order impact factors $\Phi_{A}^{(0)}$ and $\Phi_{B}^{(0)}$. Note that we have summed over the colour indices of the $t$-channel gluons implicitly. The total cross section can also be written as

$$
\sigma_{A B}^{(0)}=\frac{1}{2 s} \int\left|\mathcal{M}_{A B}^{(0)}\right|^{2} d \phi
$$

where $1 / 2 s$ denotes the flux factor (in the high energy limit), $\mathcal{M}_{A B}^{(0)}$ the matrix element for the scattering process $A+B \rightarrow A^{\prime}+B^{\prime}$ with the exchange of a gluon, and $d \phi$ stands for the phase space of the final state $A^{\prime}+B^{\prime}$. For our case of interest, $\gamma^{*} q$ (or $\gamma^{*} \gamma^{*}$ ) scattering, $A^{\prime}$ denotes a $q \bar{q}$ pair, $B^{\prime}$ a quark (or a $q \bar{q}$ pair).

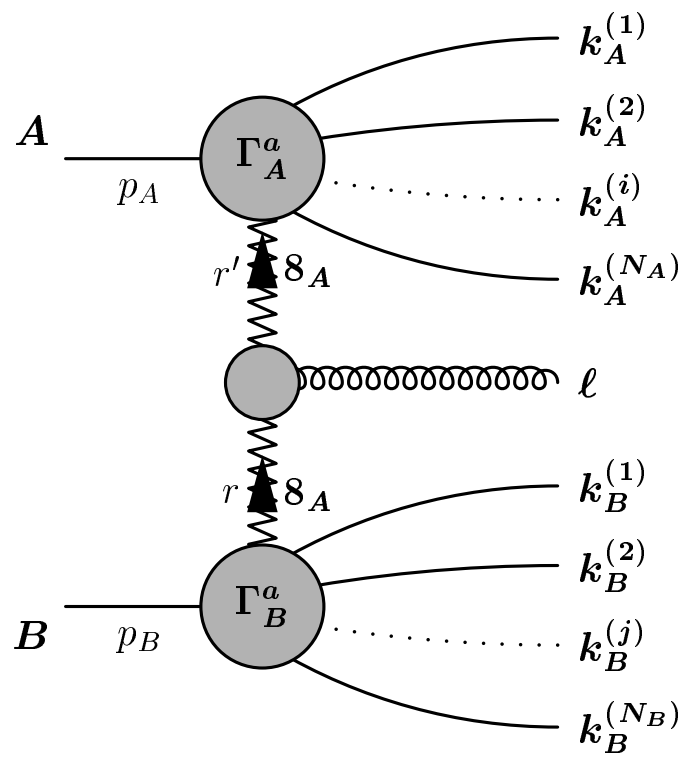

FIG. 1. Kinematics for $A+B \rightarrow A^{\prime}+g+B^{\prime}$ with initial state momenta $p_{A}, p_{B}$ and final state momenta $k_{A}^{i}, \ell, k_{B}^{j}$. An $8_{A}$-reggeon is exchanged, emitting a real gluon with momentum $\ell$ which is assumed to be separated from the other final state particles by large rapidity gaps.

In the high energy limit, the scattering amplitude $\mathcal{M}_{A B}$ for the scattering $A+B \rightarrow A^{\prime}+B^{\prime}$ (with the invariant masses of the particles or systems $A^{\prime}$ and $B^{\prime}$ being finite) (Fig. 1 w without the extra produced gluon) is described by the exchange of a reggeized gluon and can be written in the following form:

$$
\mathcal{M}_{A B}=\frac{s}{t} \Gamma_{A \rightarrow A^{\prime}}^{a}\left[\left(\frac{s}{-t}\right)^{\omega(t)}+\left(\frac{-s}{-t}\right)^{\omega(t)}\right] \Gamma_{B \rightarrow B^{\prime}}^{a} .
$$

Here, $\omega(t)$ stands for the Regge trajectory of the gluon, and $\Gamma_{A \rightarrow A^{\prime}}^{a}$ and $\Gamma_{B \rightarrow B^{\prime}}^{a}$ are the particle-particle-reggeon vertices. The index $a$ denotes the colour of the exchanged reggeized gluon. Eq.(4) exhibits the factorization property of Regge theory: when written in complex angular plane, the residue of the gluon Regge pole can be written as a product of two vertex 
functions, one for the incoming particle $A \rightarrow A^{\prime}$, the other one for $B \rightarrow B^{\prime}$. In the total cross section formula (3) this factorization property leads to the factorization of impact factors. Since (4) is valid not only in leading order, also the validity of the impact factor representation is rather general. However, when going beyond the leading order, we have to replace the $t$-channel propagators of the exchanged gluons by reggeon propagators. In leading order, we put $\omega(t)$ in (4) equal to zero and obtain

$$
\mathcal{M}_{A B}^{(0)}=\Gamma_{A \rightarrow A^{\prime}}^{(0), a} \frac{2 s}{t} \Gamma_{B \rightarrow B^{\prime}}^{(0), a} .
$$

Inserting this expression into (3), we arrive at (2).

In the next order, $\alpha_{s}^{3}$, eq.(2) receives several corrections. In order to illustrate the general pattern it is instructive to start from (3). There are three new contributions on the righthand-side of (3). The first one comes from the same intermediate state $A^{\prime}, B^{\prime}$ as in the leading order case, but there are higher order corrections inside the vertex functions $\Gamma_{A \rightarrow A^{\prime}}$ or $\Gamma_{B \rightarrow B^{\prime}}$ of the scattering amplitude $A+B \rightarrow A^{\prime}+B^{\prime}$ (or its complex conjugate). A second correction is due to the reggeization of the exchanged gluon: from (雨) it follows that in next-to-leading order reggeization provides an extra term in $\mathcal{M}_{A B}$ proportional to $\Gamma_{A \rightarrow A^{\prime}}^{(0), a}(t) \ln (s /-t) \Gamma_{B \rightarrow B^{\prime}}^{(0), a}$. These two corrections will be referred to as 'virtual' corrections to the impact factor. The third correction comes from the production of an extra gluon in the intermediate state ('real' corrections): $A+B \rightarrow A^{\prime}+g+B^{\prime}$ (Fig. 1). In the high energy limit, we divide this intermediate state into configurations with one or two large rapidity gaps: in the former case, the extra gluon belongs to the fragmentation region of $A$ or $B$, in the latter case to the central (or: multiperipheral) region.

Correspondingly, we separate the integral over the phase space $d \phi$. Begin with the configuration with one single rapidity gap. If $N_{A^{\prime}}\left(N_{B^{\prime}}\right)$ particles in the final state belong to the system $A^{\prime}\left(B^{\prime}\right)$ and carry momenta $k_{A^{\prime}}^{i}\left(k_{B^{\prime}}^{j}\right)$, we may write the phase space in $D$ dimensions explicitly as

$$
d \phi=d \tilde{\phi}_{A^{\prime}} d \tilde{\phi}_{B^{\prime}}(2 \pi)^{D} \delta^{(D)}\left(p_{A}+p_{B}-\sum_{i} k_{A^{\prime}}^{i}-\sum_{j} k_{B^{\prime}}^{j}\right)
$$

with

$$
d \tilde{\phi}_{A^{\prime}}=\prod_{i=1}^{N_{A}} \frac{d^{D} k_{A^{\prime}}^{i}}{(2 \pi)^{D-1}} \delta^{+}\left(k_{A^{\prime}}^{i}{ }^{2}-m_{A}^{i 2}\right)
$$

and

$$
d \tilde{\phi}_{B^{\prime}}=\prod_{j=1}^{N_{B}} \frac{d^{D} k_{B^{\prime}}^{j}}{(2 \pi)^{D-1}} \delta^{+}\left(k_{B^{\prime}}^{j}{ }^{2}-m_{B}^{j 2}\right) .
$$

Introducing the explicit integration over the reggeon momentum $r$ with a Sudakov decomposition $r=\alpha_{r} q^{\prime}+\beta_{r} p+r_{\perp}$ via one extra delta function and

$$
\frac{d^{D} r}{(2 \pi)^{D}}=\frac{s}{2} \frac{d \alpha_{r}}{2 \pi} \frac{d \beta_{r}}{2 \pi} \frac{d^{D-2} \boldsymbol{r}}{(2 \pi)^{D-2}}
$$


we may write

$$
d \phi=d \phi_{A^{\prime}} \frac{s d \beta_{r}}{2 \pi} d \phi_{B^{\prime}} \frac{s d \alpha_{r}}{2 \pi} \frac{1}{2 s} \frac{d^{D-2} \boldsymbol{r}}{(2 \pi)^{D-2}},
$$

where $d \phi_{A^{\prime}}=d \tilde{\phi}_{A^{\prime}}(2 \pi)^{D} \delta^{(D)}\left(p_{A}+r-\sum_{i} k_{A^{\prime}}^{i}\right)$ and $d \phi_{B^{\prime}}=(2 \pi)^{D} d \tilde{\phi}_{B^{\prime}} \delta^{(D)}\left(p_{B}-r-\sum_{j} k_{B^{\prime}}^{j}\right)$ are the usual phase space measures for the final state particles belonging to the systems $A^{\prime}$ and $B^{\prime}$ respectively. For an intermediate state with two rapidity gaps (gluon in the central region) we have two $t$-channel reggeon momenta, $r$ and $r^{\prime}$. Denoting the momentum of the produced gluon by $\ell \equiv r-r^{\prime}=\alpha_{\ell} q^{\prime}+\beta_{\ell} p+\ell_{\perp}$ eq. (10) will be generalized to:

$$
d \phi=\left(\frac{1}{2 s}\right)^{2} d \phi_{A^{\prime}} \frac{s d \beta_{r}}{2 \pi} \frac{s d \alpha_{r}}{2 \pi} d \phi_{g} \frac{s d \beta_{r^{\prime}}}{2 \pi} \frac{s d \alpha_{r^{\prime}}}{2 \pi} d \phi_{B^{\prime}} \frac{d^{D-2} \boldsymbol{r}}{(2 \pi)^{D-2}} \frac{d^{D-2} \boldsymbol{r}^{\prime}}{(2 \pi)^{D-2}}
$$

where

$$
d \phi_{g}=\frac{d^{D} \ell}{(2 \pi)^{D-1}} \delta^{+}\left(\ell^{2}\right)(2 \pi)^{D} \delta^{(D)}\left(r-r^{\prime}+\ell\right)
$$

is the phase space measure for the gluon in the central region.

Returning to the impact factor representation (2), the NLO corrections due to the extra gluon in the $s$-channel can be grouped into two parts. The gluon in the fragmentation region of $A$ or $B$ leads to corrections of the impact factor $\Phi_{A}$ or $\Phi_{B}$, respectively, whereas the gluon in the central region represents the first rung of the BFKL ladder (in the leading-logarithmic approximation) [3]. Together with the other NLO corrections mentioned before, the entire sum of NLO corrections to the total cross section can be cast into the following form:

$$
\begin{aligned}
\sigma_{A B}^{(1)}= & \frac{1}{s} \operatorname{Im} T_{A B}^{(1)}(s, t=0) \\
= & \int \frac{d^{D-2} \boldsymbol{r}}{(2 \pi)^{D-2}} \Phi_{A}^{(1)} \frac{1}{\boldsymbol{r}^{4}} \Phi_{B}^{(0)}+\int \frac{d^{D-2} \boldsymbol{r}}{(2 \pi)^{D-2}} \Phi_{A}^{(0)} \frac{1}{\boldsymbol{r}^{4}} \Phi_{B}^{(1)} \\
& +\int \frac{d^{D-2} \boldsymbol{r}}{(2 \pi)^{D-2}} \Phi_{A}^{(0)} \ln \left(s / \boldsymbol{r}^{2}\right) 2 \omega^{(1)}\left(\boldsymbol{r}^{2}\right) \frac{1}{\boldsymbol{r}^{4}} \Phi_{B}^{(0)} \\
& +\ln \left(s / s_{0}\right) \int \frac{d^{D-2} \boldsymbol{r}}{(2 \pi)^{D-2}} \frac{d^{D-2} \boldsymbol{r}^{\prime}}{(2 \pi)^{D-2}} \Phi_{A}^{(0)} \frac{1}{\boldsymbol{r}^{4}} \mathcal{K}_{\text {real }}\left(\boldsymbol{r}, \boldsymbol{r}^{\prime}\right) \frac{1}{\boldsymbol{r}^{\prime 4}} \Phi_{B}^{(0)} .
\end{aligned}
$$

Here $\mathcal{K}_{\text {real }}\left(\boldsymbol{r}, \boldsymbol{r}^{\prime}\right)$ denotes the square of the gluon production vertex, the BFKL kernel (sometimes referred to as the 'real' part of the BFKL kernel, i.e. it does not contain the gluon reggeization). Later on it will be convenient to rewrite the reggeization of the gluon (third term on the right-hand-side): putting $\ln \left(s / \boldsymbol{r}^{2}\right)=\ln \left(s / s_{0}\right)+\ln \left(s_{0} / \boldsymbol{r}^{2}\right)$ we combine the $\ln \left(s / s_{0}\right)$ piece with the real part of the BFKL kernel: in the sum of both terms the infrared singularity in the limit $\boldsymbol{r}-\boldsymbol{r}^{\prime} \rightarrow 0$ drops out. Note the factorization of eq. (13): as indicated after (4), this feature is a general consequence of Regge theory and (with suitable generalizations of the $t$-channel gluon propagators) is expected to hold in arbitrary order perturbation theory. For the impact factor we state the general definition:

$$
\Phi_{A}=\frac{\delta^{a b}}{\sqrt{N_{c}^{2}-1}} \sum_{A^{\prime}} \int\left\langle\Gamma_{A \rightarrow A^{\prime}}^{a} \Gamma_{A \rightarrow A^{\prime}}^{b *}\right\rangle d \phi_{A^{\prime}} \frac{s d \beta_{r}}{2 \pi}
$$


The average symbol \langle\rangle stands for the sum over color and helicity in the intermediate state $A^{\prime}$. In the following we will use the short hand notation

$$
\Phi_{A}=\sum_{A^{\prime}} \int\left|\Gamma_{A \rightarrow A^{\prime}}\right|^{2} d \phi_{A^{\prime}} \frac{s d \beta_{r}}{2 \pi},
$$

In particular, in the square of the matrix elements we have included the colour projector $\delta^{a b} / \sqrt{N_{c}^{2}-1}$.

In this paper we are interested in the NLO corrections to the photon impact factor. The strategy follows from the discussion outlined in this section. The NLO corrections can be divided into the $q \bar{q}$ and $q \bar{q} g$ intermediate states (named virtual and real corrections, respectively). In the former case we need the NLO corrections to the $\gamma^{*} \rightarrow q \bar{q}$ vertex, $\Gamma_{\gamma^{*} \rightarrow q \bar{q}}^{(1)}$. They contain infrared divergences (in dimensional regularization: poles in $\epsilon$ ) and are listed in [1]. An additional (infrared divergent) contribution comes from the gluon trajectory function in the third term on the right-hand-side of (13). For the $q \bar{q} g$ contribution we need the vertex $\Gamma_{\gamma^{*} \rightarrow q \bar{q} g}$, in the Born approximation: since, by definition, the gluon lies in the fragmentation region of the virtual photon, we have to divide the phase space of the produced gluon. In section VI we present a detailed discussion of this rather subtle issue: we will show that the separation of the fragmentation region introduces the scale $s_{0}$, in the last term of (13). The fragmentation region contains soft and collinear divergences. When combining them with the infrared divergences of the $q \bar{q}$ state (including those from the gluon trajectory function),

the infrared divergences cancel [4]. It is the main purpose of this paper to find a separation of the fragmentation from the central region which allows to define infrared finite combinations of virtual and real corrections to the impact factor.

\section{SINGULAR TERMS OF THE VIRTUAL CORRECTIONS}

For future reference, and in order to define our notation we briefly summarize the Born level impact factor and list the divergent terms of the virtual corrections. The amplitude $T_{\gamma^{*} q}$ for the scattering $\gamma^{*}+q^{\prime} \rightarrow q \bar{q}+q^{\prime}$ (Fig. 2) has been calculated in [1] to next-to-leading order in $\alpha_{s}$. We write $T_{\gamma^{*} q}$ as an expansion in the strong coupling $g$

$$
T_{\gamma^{*} q}=g^{2} T_{\gamma^{*} q}^{(0)}+g^{4} T_{\gamma^{*} q}^{(1)} .
$$

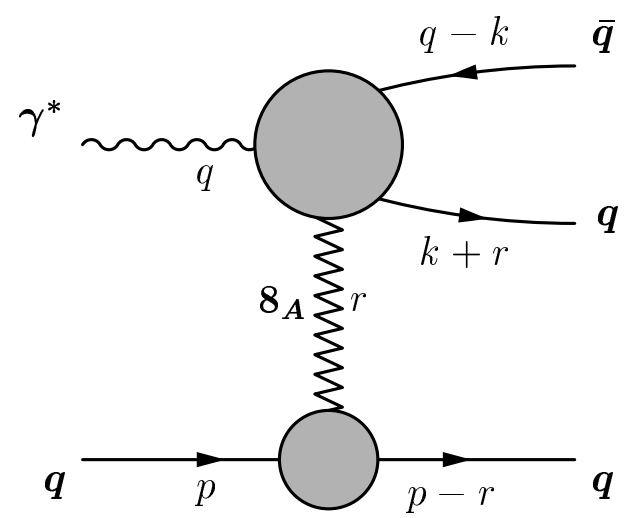

FIG. 2. Kinematics of the process $\gamma^{*}+q^{\prime} \rightarrow q \bar{q}+q^{\prime}$. 
The momenta are labelled as in Fig. 2. The kinematical variables we use are the centreof-mass energy $s$ of the incoming virtual photon and the incoming quark, the virtuality of the photon, $Q^{2}$, the Bjorken variable $x=Q^{2} / 2 p \cdot q$, the momentum transfers $t=r^{2}, t_{a}=k^{2}$, $t_{b}=(q-k-r)^{2}$, and the invariant mass $M^{2}$ of the outgoing $q \bar{q}$-system. In addition we use a Sudakov decomposition of the momentum $k$ with respect to the light cone momenta $q^{\prime}=q-x p$ and $p$ with $2 p \cdot q^{\prime}=s, k=\alpha q^{\prime}+\beta p+k_{\perp}$. Two dimensional transverse momenta are denoted as $\boldsymbol{k}^{2}=-k_{\perp}^{2}$.

The Born impact factor is obtained from $T_{\gamma^{*} q}^{(0)}$. To leading order we may write

$$
T_{\gamma^{*} q}^{(0)}=\Gamma_{\gamma^{*} \rightarrow q \bar{q}}^{(0), a} \frac{2 s}{t} \Gamma_{q q}^{(0), a}
$$

with the Born level vertices

$$
\begin{aligned}
\Gamma_{q q}^{(0), a} & =\frac{1}{s} \bar{u}\left(p-r, \lambda_{q^{\prime}}\right) \not q^{\prime} \lambda^{a} u\left(p, \lambda_{q}\right), \\
\Gamma_{\gamma^{*} \rightarrow q \bar{q}}^{(0), a} & =-i e e_{f}\left(\frac{H_{T}^{a}}{s t_{a}}-\frac{\bar{H}_{T}^{a}}{s t_{b}}\right),
\end{aligned}
$$

where

$$
\begin{aligned}
& H_{T}^{a}=\bar{u}(k+r, \lambda) \not p \not k \not \lambda^{a} v\left(q-k, \lambda^{\prime}\right), \\
& \bar{H}_{T}^{a}=\bar{u}(k+r, \lambda) \not \notin(\not q-\not k-\not \gamma) \not p \lambda^{a} v\left(q-k, \lambda^{\prime}\right)
\end{aligned}
$$

and $\lambda^{a}$ are the generators of the colour group $S U\left(N_{c}\right)$. Squaring the Born level vertices (18) and averaging (summing) over incoming (outgoing) colours and helicities we obtain for the coupling to the reggeized gluons with colours $(a, b)$, contracted with the colour singlet projector (cf. eqs. (14) and (15)):

$$
\left|\Gamma_{q q}^{(0)}\right|^{2}=\frac{\sqrt{N_{c}^{2}-1}}{2 N_{c}}
$$

The $\gamma^{*} \rightarrow q \bar{q}$-vertex $\left|\Gamma_{\gamma^{*} \rightarrow q \bar{q}}^{(0)}\right|^{2}=\left|\Gamma_{\gamma^{*} \rightarrow q \bar{q}}^{(0)}(\boldsymbol{k}, \alpha)\right|^{2}$ for a longitudinally polarized $\gamma^{*}$ coupling to a quark flavour $f$ reads

$$
\left|\Gamma_{\gamma^{*} \rightarrow q \bar{q}}^{(0)}\right|_{L}^{2}=4 \sqrt{N_{c}^{2}-1} e^{2} e_{f}^{2} \alpha^{3}(1-\alpha)^{3} Q^{2}\left(\frac{1}{D(\boldsymbol{k})}-\frac{1}{D(\boldsymbol{k}+\boldsymbol{r})}\right)^{2},
$$

where $D(\boldsymbol{k})=\boldsymbol{k}^{2}+\alpha(1-\alpha) Q^{2}$. Here the exchanged reggeized gluons must be in the colour singlet. For the sum over the two transverse photon polarizations we have

$$
\left|\Gamma_{\gamma^{*} \rightarrow q \bar{q}}^{(0)}\right|_{T}^{2}=2 \sqrt{N_{c}^{2}-1} e^{2} e_{f}^{2} \alpha(1-\alpha)\left[\alpha^{2}+(1-\alpha)^{2}\right]\left(\frac{\boldsymbol{k}}{D(\boldsymbol{k})}-\frac{\boldsymbol{k}+\boldsymbol{r}}{D(\boldsymbol{k}+\boldsymbol{r})}\right)^{2} .
$$

The LO impact factor follows from the definition in eq. (15), from the two-particle phase space measure

$$
d \phi_{q \bar{q}} \frac{s d \beta_{r}}{2 \pi}=\frac{d \alpha}{2 \alpha(1-\alpha)} \frac{d^{D-2} \boldsymbol{k}}{(2 \pi)^{D-1}}
$$


and from the Born level vertices (23, 24). Expanding $\Phi_{\gamma^{*}}$ in the strong coupling,

$$
\Phi_{\gamma^{*}}=g^{2} \Phi_{\gamma^{*}}^{(0)}+g^{4} \Phi_{\gamma^{*}}^{(1)}
$$

we write the Born level impact factor as

$$
\Phi_{\gamma^{*} ; T, L}^{(0)}=\int \frac{d^{D-2} \boldsymbol{k}}{(2 \pi)^{D-1}} \frac{d \alpha}{2 \alpha(1-\alpha)}\left|\Gamma_{\gamma^{*} \rightarrow q \bar{q}}^{(0)}\right|_{T, L}^{2} \equiv \int d^{D-2} \boldsymbol{k} d \alpha \mathcal{I}_{2 ; T, L}(\alpha, \boldsymbol{k} ; \boldsymbol{r}, Q) .
$$

The last equation defines the integrand of the LO impact factor:

$$
\begin{gathered}
\mathcal{I}_{2 ; L}(\alpha, \boldsymbol{k} ; \boldsymbol{r}, Q)=\frac{2 e^{2} e_{f}^{2} \sqrt{N_{c}^{2}-1}}{(2 \pi)^{D-1}} \alpha^{2}(1-\alpha)^{2} Q^{2}\left(\frac{1}{D(\boldsymbol{k})}-\frac{1}{D(\boldsymbol{k}+\boldsymbol{r})}\right)^{2} \\
\mathcal{I}_{2 ; T}(\alpha, \boldsymbol{k} ; \boldsymbol{r}, Q)=\frac{e^{2} e_{f}^{2} \sqrt{N_{c}^{2}-1}}{(2 \pi)^{D-1}}\left[\alpha^{2}+(1-\alpha)^{2}\right]\left(\frac{\boldsymbol{k}}{D(\boldsymbol{k})}-\frac{\boldsymbol{k}+\boldsymbol{r}}{D(\boldsymbol{k}+\boldsymbol{r})}\right)^{2}
\end{gathered}
$$

Because of the symmetry of the squared matrix element under the exchange $q \leftrightarrow \bar{q}$, namely $\alpha \leftrightarrow 1-\alpha, \boldsymbol{k} \leftrightarrow-\boldsymbol{r}-\boldsymbol{k}$, we have the relation $\mathcal{I}_{2}(\alpha, \boldsymbol{k} ; \boldsymbol{r}, Q)=\mathcal{I}_{2}(1-\alpha,-\boldsymbol{r}-\boldsymbol{k} ; \boldsymbol{r}, Q)$.

Turning to the NLO part of (16), we split $T_{\gamma^{*} q}$ into a finite part $F$ and a divergent part $D$ as

$$
T_{\gamma^{*} q}^{(1)}=F_{\gamma^{*} q}+\frac{c_{\Gamma}}{(4 \pi)^{2-\epsilon}} T_{\gamma^{*} q}^{(0)} D, \quad c_{\Gamma} \equiv \frac{\Gamma(1+\epsilon) \Gamma^{2}(1-\epsilon)}{\Gamma(1-2 \epsilon)} .
$$

The divergences are given in dimensional regularization and appear as poles when the spacetime dimension $D=4-2 \epsilon$ approaches $D=4$. Here we explicitly include the divergent contribution from self-energy insertions on all four external quark lines

$$
D_{\mathrm{SE}}=-2 C_{F}\left(\frac{1}{\epsilon_{\mathrm{UV}}}-\frac{1}{\epsilon}\right)
$$

labelling poles from ultraviolet divergences explicitly by $\epsilon_{\mathrm{UV}}$. As usual, the $S U\left(N_{c}\right)$ invariants are denoted by $C_{A}=N_{c}$ and $C_{F}=\left(N_{c}^{2}-1\right) /\left(2 N_{c}\right)$.

The divergent part can then be written as the sum of three terms

$$
D=D_{\gamma^{*} \rightarrow q \bar{q}}+D_{\omega}+D_{q q},
$$

following the expansion of the Regge ansatz (四) in powers of $\alpha_{s}$. The three terms denote the divergent parts of the vertex $\Gamma_{\gamma^{*} \rightarrow q \bar{q}}^{(1)}$, of the Regge trajectory of the gluon, $\omega^{(1)}(t)$, which is accompanied by the large leading logarithms in $s$, and of the vertex $\Gamma_{q q}^{(1)}$, respectively. They have the form:

$$
\begin{gathered}
D_{\gamma^{*} \rightarrow q \bar{q}}=-\frac{2 C_{F}}{\epsilon^{2}}-\frac{3 C_{F}}{\epsilon}+\frac{\beta_{0}}{\epsilon_{\mathrm{UV}}}+\frac{2 C_{F}}{\epsilon} \ln \left(-M^{2}\right)+\frac{C_{A}}{\epsilon}\left(\ln \frac{\alpha(1-\alpha) t}{M^{2}}+\ln \frac{s_{0}}{-t}\right), \\
D_{\omega}=\frac{C_{A}}{\epsilon}\left[\ln \frac{s}{s_{0}}+\ln \frac{-s}{s_{0}}\right]
\end{gathered}
$$


and

$$
D_{q q}=-\frac{2 C_{F}}{\epsilon^{2}}-\frac{3 C_{F}}{\epsilon}+\frac{\beta_{0}}{\epsilon_{\mathrm{UV}}}+\frac{2 C_{F}}{\epsilon} \ln (-t)+\frac{C_{A}}{\epsilon} \ln \frac{s_{0}}{-t} .
$$

Here, we have separated infrared and ultraviolet divergences, making use of the formulae given in [1]. Note that, compared to the Regge ansatz (4), we have changed the energy scale. In (34), we have introduced the energy scale $s_{0}$ : instead of $\ln s /(-t)$ we write $\ln s /(-t)=$ $\ln s / s_{0}+\ln s_{0} /(-t)$ and absorb the second term into $D_{\gamma^{*} \rightarrow q \bar{q}}$ and $D_{q q}$ in (33, 35). Let us first consider the infrared singular terms. For the virtual NLO corrections to the photon impact factor we start from

$$
\Phi_{\gamma^{*}}^{(1, \text { virtual })}=\frac{\delta_{a b}}{\sqrt{N_{c}^{2}-1}} \int \frac{d^{D-2} \boldsymbol{k}}{(2 \pi)^{D-1}} \frac{d \alpha}{2 \alpha(1-\alpha)} \Gamma_{\gamma^{*} \rightarrow q \bar{q}}^{(1), a} \Gamma_{\gamma^{*} \rightarrow q \bar{q}}^{(0), b *} d \phi_{q \bar{q}} \frac{s d \beta_{r}}{2 \pi}+\text { c.c. . }
$$

Making use of eqs. (30) and (33) - the latter one without the UV pole which we will discuss later - we can immediately deduce the IR divergent part of the virtual correction to the impact factor:

$$
\begin{aligned}
\left.\Phi_{\gamma^{*}}^{(1, \text { virtual })}\right|^{\text {IRdivergent }}=\frac{c_{\Gamma}}{(4 \pi)^{2-\epsilon}} \Phi_{\gamma^{*}}^{(0)}\left[D_{\gamma^{*} \rightarrow q \bar{q}}^{\mathrm{IR}}+\text { c.c. }\right] \\
=\int d \boldsymbol{k} \int_{0}^{1} d \alpha \mathcal{I}_{2}(\alpha, \boldsymbol{k} ; \boldsymbol{r}, Q) \frac{c_{\Gamma}}{(4 \pi)^{2-\epsilon}} \\
\quad \times\left\{\frac{C_{A}}{\epsilon}\left[2 \log s_{0}+2 \log \alpha(1-\alpha)-2 \log M^{2}\right]+\frac{C_{F}}{\epsilon}\left[-\frac{4}{\epsilon}+4 \log M^{2}-6\right]\right\} .
\end{aligned}
$$

Below we will show that these divergent terms cancel against the real corrections.

Turning to the ultraviolet divergent pieces in $D_{\gamma^{*} \rightarrow q \bar{q}}$ and $D_{q q}$, they are proportional to $\beta_{0}=\left(11 N_{c}-2 n_{f}\right) / 6$, and in [1] it has been shown that they are removed by the renormalization of the strong coupling. It is instructive to repeat the argument in more detail. The ultraviolet part of (33), including constants, is obtained by adding, in [1], eqs. (56), (57), (61), (62), (67), plus one half of eq. (60):

$$
\frac{g^{4} c_{\Gamma}}{(4 \pi)^{2-\epsilon}} \Gamma_{\gamma^{*} \rightarrow q \bar{q}}^{(0), a}\left[\frac{\left(\boldsymbol{r}^{2}\right)^{-\epsilon}}{\epsilon_{U V}}\left(\frac{14 N_{c}}{6}-\frac{1}{3} n_{f}-\frac{1}{2 N_{c}}\right)-\frac{C_{F}}{\epsilon_{U V}}\right]
$$

Except for the last term which is due to the wave function renormalization of the massless outgoing quarks, all ultraviolet poles are proportional to $\left(\boldsymbol{r}^{2}\right)^{-\epsilon}$. It will be convenient to slightly modify the last term: instead of using, for the renormalization of the outgoing massless quarks, the decomposition (31), we write

$$
D_{\mathrm{SE}}^{\prime}=-2 C_{F}\left(\frac{\left(\boldsymbol{r}^{2}\right)^{-\epsilon}}{\epsilon_{\mathrm{UV}}}-\frac{\left(\boldsymbol{r}^{2}\right)^{-\epsilon}}{\epsilon}\right) .
$$

This allows to simplify (38):

$$
\frac{g^{4} c_{\Gamma}}{(4 \pi)^{2-\epsilon}} \Gamma_{\gamma^{*} \rightarrow q \bar{q}}^{(0), a} \frac{\left(\boldsymbol{r}^{2}\right)^{-\epsilon}}{\epsilon_{U V}} \beta_{0}
$$


When addressing, in the next step of our program, the calculation of the finite terms of the virtual corrections, we will have to remember that the change (39) in the wave function renormalization introduces a new finite term

$$
-\frac{g^{4}}{(4 \pi)^{2}} \Gamma_{\gamma^{*} \rightarrow q \bar{q}}^{(0), a} C_{F} \ln \left(\boldsymbol{r}^{2}\right)
$$

In order to remove the UV divergences we have to renormalize, i.e. we add the Born approximation with the replacement:

$$
g \rightarrow \frac{Z_{1} g_{\mu} \mu^{\epsilon}}{Z_{2} \sqrt{Z_{3}}}=g_{\mu} \mu^{\epsilon}\left[1-\frac{\alpha_{s}}{4 \pi} \beta_{0}\left(\frac{1}{\epsilon_{U V}}-\gamma_{E}+\ln (4 \pi)\right)+\ldots\right]
$$

with the renormalization constants taken in the $\overline{\mathrm{MS}}$ scheme. When both contributions are added, the ultraviolet poles cancel, and we are left with

$$
g^{2} \Gamma_{\gamma^{*} \rightarrow q \bar{q}}^{(1 ; \mathrm{UV})}=-\frac{\alpha_{s}}{4 \pi} \Gamma_{\gamma^{*} \rightarrow q \bar{q}}^{(0)} \beta_{0} \ln \frac{\boldsymbol{r}^{2}}{\mu^{2}} .
$$

Note that all dependence upon the renormalization scale $\mu$ is contained in the logarithm which introduces the scale dependence of the strong coupling constant, $\alpha_{s}\left(\boldsymbol{r}^{2}\right)$. Correspondingly, in the photon impact factor the $\mu$-dependence is contained in the contribution:

$$
g^{2} \Phi_{\gamma^{*}}^{(1 ; \mathrm{UV})}=-\frac{\alpha_{s}}{2 \pi} \Phi_{\gamma^{*}}^{(0)} \beta_{0} \ln \frac{\boldsymbol{r}^{2}}{\mu^{2}}
$$

as it is required by the renormalization group equation. Our derivation might suggest that $\boldsymbol{r}^{2}$ is the 'natural' momentum scale of $\alpha_{s}$ in the photon impact factor: however, it is clear that we can write

$$
g^{2} \Phi_{\gamma^{*}}^{(1 ; \mathrm{UV})}=-\frac{\alpha_{s}}{2 \pi} \Phi_{\gamma^{*}}^{(0)}\left(\beta_{0} \ln \frac{Q^{2}}{\mu^{2}}+\beta_{0} \ln \frac{\boldsymbol{r}^{2}}{Q^{2}}\right)
$$

i.e. by simply redefining the $\mu$-independent part of the impact factor we can switch from one momentum scale of $\alpha_{s}, \alpha_{s}\left(\boldsymbol{r}^{2}\right)$, to another scale, $\alpha_{s}\left(Q^{2}\right)$.

Stating that (44) is the only $\mu$-dependent term in the impact factor implies that the $\mu$-dependence drops out of all the remaining contributions to the impact factor. In fact, we silently dropped a $\mu$ already in eq. (41), anticipating the scale invariance of the finite terms which are left after the cancellation of the infrared divergencies of real and virtual corrections. This can be understood as follows. Without having it written explicitly in [1], the infrared divergences in dimensional regularization appear as poles in $\epsilon$, accompanied by the power of some dimensionful scale, $s_{i}$. In a one-loop calculation the sum of infrared divergent terms from real $(\mathrm{R})$ and virtual $(\mathrm{V})$ corrections generally reads

$$
T_{\mathrm{IR}}=\sum_{i} A_{i} \frac{\left(s_{i}\right)^{-\epsilon}}{\epsilon^{2}}+B_{i} \frac{\left(s_{i}\right)^{-\epsilon}}{\epsilon}
$$

Adding real and virtual corrections, the coefficients $A_{i}$ and $B_{i}$ are sums as well,

$$
A_{i}=A_{i}^{V}+A_{i}^{R}, \quad B_{i}=B_{i}^{V}+B_{i}^{R} .
$$


Expanding (46) in $\epsilon$ we obtain

$$
T_{\mathrm{IR}}=\sum_{i} \frac{A_{i}}{\epsilon^{2}}-\frac{A_{i}}{\epsilon} \ln s_{i}+\frac{A_{i}}{2} \ln ^{2} s_{i}+\frac{B_{i}}{\epsilon}-B_{i} \ln s_{i} .
$$

Introducing the scale $\mu$ with the renormalized coupling as a factor $\mu^{2 \epsilon}$ and reexpanding results in

$$
\begin{aligned}
T_{\mathrm{IR}}= & \sum_{i}\left(\frac{1}{\epsilon^{2}}+\frac{\ln \mu^{2}}{\epsilon}+\frac{\ln ^{2} \mu^{2}}{2}\right) A_{i}-\left(\frac{1}{\epsilon}+\ln \mu^{2}\right) A_{i} \ln s_{i}+\frac{A_{i}}{2} \ln ^{2} s_{i} \\
& +\left(\frac{1}{\epsilon}+\ln \mu^{2}\right) B_{i}-B_{i} \ln s_{i} .
\end{aligned}
$$

Further below (section VII) we will show that in the sum of virtual and real corrections all $\epsilon$-poles cancel: this means that $A_{i}=0$ and $\sum_{i} B_{i}=0$. Hence, we see that the cancellation of infrared divergences implies that also the $\ln \mu^{2}$ terms cancel. This allows us to write e.g. the logarithm in (41) with a dimensionful argument. We did the same with the results of diagram 14 in [1] after expanding divergent terms of the form $s_{i}^{-\epsilon}$.

\section{REAL CORRECTIONS}

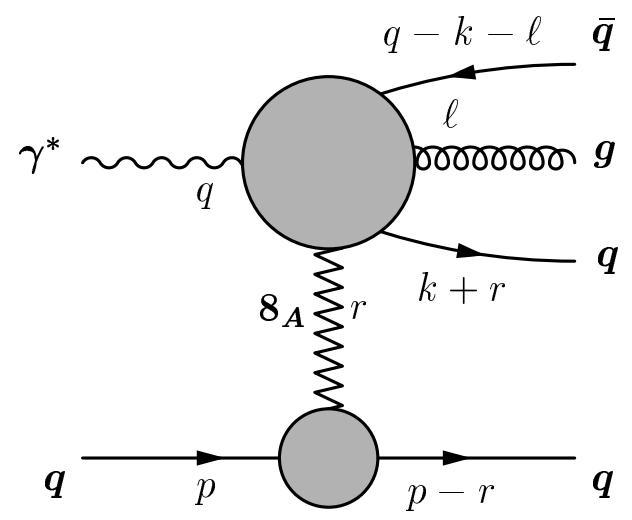

FIG. 3. Kinematics of the process $\gamma^{*}+q^{\prime} \rightarrow q \bar{q} g+q^{\prime}$.

We now turn to the real corrections. We study the process $\gamma^{*}+q \rightarrow(q \bar{q} g)+q$ with transverse photon polarization (Fig.33); the case of the longitudinal photon has been studied in [2], and we follow the procedure outlined in this earlier paper. The diagrams are shown in Fig. 1 . We use the Sudakov decomposition of the gluon momentum $\ell=\alpha_{\ell} q^{\prime}+\beta_{\ell} p+\ell_{\perp}$ and introduce the following abbreviations :

$$
\begin{aligned}
\alpha_{1} & \equiv \alpha, \\
\bar{\alpha}_{1} & \equiv\left(1-\alpha-\alpha_{\ell}\right), \\
\alpha_{2} & \equiv(1-\alpha), \\
\bar{\alpha}_{2} & \equiv\left(\alpha+\alpha_{\ell}\right) .
\end{aligned}
$$



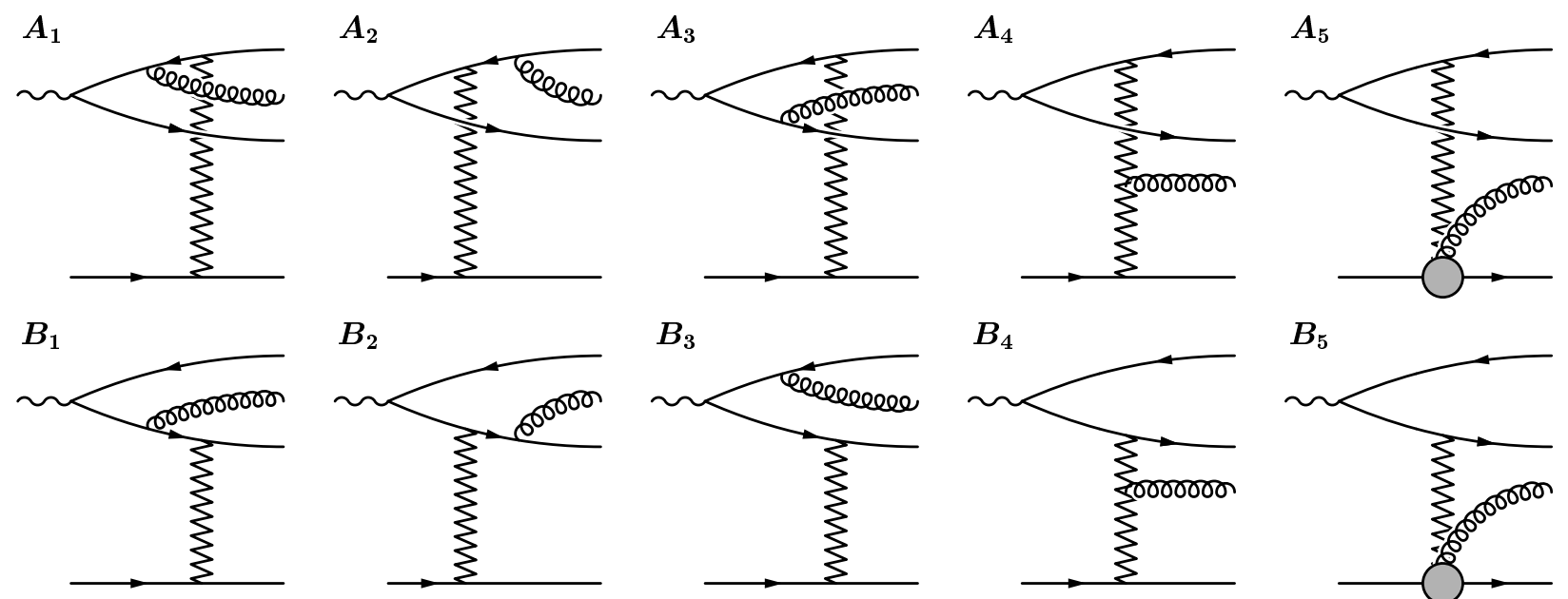

FIG. 4. Feynman diagrams for $\gamma^{*}+q^{\prime} \rightarrow q \bar{q} g+q^{\prime}$.

The propagator denominators, that occur in the calculation, have the following form:

$$
\begin{aligned}
D_{1} & =(k+\ell+r-q)^{2} \\
& =-\bar{\alpha}_{1} Q^{2}-(\boldsymbol{k}+\boldsymbol{\ell}+\boldsymbol{r})^{2}-\frac{\bar{\alpha}_{1}}{\alpha_{1}}(\boldsymbol{k}+\boldsymbol{r})^{2}-\frac{\bar{\alpha}_{1}}{\alpha_{\ell}} \ell^{2} \\
D_{2} & =(k+r-q)^{2} \\
& =-\alpha_{2} Q^{2}-\frac{1}{\alpha_{1}}(\boldsymbol{k}+\boldsymbol{r})^{2} \\
D_{3} & =(k+\ell+r)^{2} \\
& =-(\boldsymbol{k}+\boldsymbol{\ell}+\boldsymbol{r})^{2}+\frac{\bar{\alpha}_{2}}{\alpha_{1}}(\boldsymbol{k}+\boldsymbol{r})^{2}+\frac{\bar{\alpha}_{2}}{\alpha_{\ell}} \ell^{2} \\
& =\frac{1}{\alpha_{\ell} \alpha_{1}}\left(-\alpha_{\ell}(\boldsymbol{k}+\boldsymbol{r})+\alpha_{1} \ell\right)^{2} \\
D_{4} & =(k-q)^{2} \\
& =-\boldsymbol{k}^{2}+\frac{\alpha_{2}}{\bar{\alpha}_{1}}(\boldsymbol{k}+\boldsymbol{\ell})^{2}+\frac{\alpha_{2}}{\alpha_{\ell}} \ell^{2} \\
& =\frac{1}{\alpha_{\ell} \bar{\alpha}_{1}}\left(\alpha_{\ell}(\boldsymbol{k}+\boldsymbol{\ell})+\bar{\alpha}_{1} \ell\right)^{2} \\
D_{5} & =(\ell-r)^{2} \\
& =-\alpha_{\ell} Q^{2}-\frac{\alpha_{\ell}}{\alpha_{1}}(\boldsymbol{k}+\boldsymbol{r})^{2}-\frac{\alpha_{\ell}}{\bar{\alpha}_{1}}(\boldsymbol{k}+\boldsymbol{\ell})^{2}-(\boldsymbol{\ell}-\boldsymbol{r})^{2} \\
D_{6} & =k^{2} \\
& =-\alpha_{1} Q^{2}-\boldsymbol{k}^{2}-\frac{\alpha_{1}}{\bar{\alpha}_{1}}(\boldsymbol{k}+\boldsymbol{\ell})^{2}-\frac{\alpha_{1}}{\alpha_{\ell}} \ell^{2} \\
D_{7} & =(k+\ell)^{2} \\
& =-\bar{\alpha}_{2} Q^{2}-\frac{1}{\bar{\alpha}_{1}}(\boldsymbol{k}+\boldsymbol{\ell})^{2} .
\end{aligned}
$$


We present the results for $\left|\mathcal{M}_{q \bar{q} g}\right|^{2}$, the squared matrix element, averaged (summed) over the helicities and colors of the incoming (outgoing) quarks and summed over equal photon polarizations; thereby we made use of $\sum_{i=j=1,2}\left(\epsilon_{T}^{i} k_{\perp}\right)\left(\epsilon_{T}^{j} \ell_{\perp}\right)=\boldsymbol{k} \boldsymbol{\ell}$. Our results can be simplified by expressing them in terms of the matrix elements for longitudinal photons; for this reason we also recall the results for longitudinal photon polarization [2]. The matrix elements for the process $\gamma^{*} q \rightarrow(q \bar{q} g) q$ can be written in the following factorized form:

$$
\left|\mathcal{M}_{q \bar{q} g}\right|^{2}=\frac{1}{N_{c}^{2}-1} g^{2} \delta^{a b}\left|\Gamma_{\gamma^{*} \rightarrow q \bar{q} g}^{(0)}\right|^{2}\left(\frac{2 s}{t}\right)^{2} \delta^{a b}\left|\Gamma_{q q}^{(0)}\right|^{2},
$$

and by using eq.(22) we extract the squared vertex $\left|\Gamma_{\gamma^{*} \rightarrow q \bar{q} g}^{(0)}\right|^{2}$ (summed over helicities and color in the $q \bar{q} g$ state). Following the notation used in [2] we list our results in correspondence with the Feynman diagrams:

$$
\begin{aligned}
& \left|\Gamma_{\gamma^{*} \rightarrow q \bar{q} g}^{(0)}\right|_{L}^{2}=4 e^{2} e_{f}^{2} g^{4} \sqrt{N_{c}^{2}-1} Q^{2} \sum_{i, j=1}^{5}\left(\mathcal{A A}_{i j}^{L}+\mathcal{A B}_{i j}^{L}+\mathcal{B} \mathcal{A}_{i j}^{L}+\mathcal{B B}_{i j}^{L}\right) \\
& \left|\Gamma_{\gamma^{*} \rightarrow q \bar{q} g}^{(0)}\right|_{T}^{2}=e^{2} e_{f}^{2} g^{4} \sqrt{N_{c}^{2}-1} \sum_{i, j=1}^{5}\left(\mathcal{A}_{i j}^{T}+\mathcal{A B}_{i j}^{T}+\mathcal{B} \mathcal{A}_{i j}^{T}+\mathcal{B B}_{i j}^{T}\right) \\
& \mathcal{A A}_{11}^{T}=C_{F} \frac{4}{D_{1}{ }^{2} D_{2}{ }^{2}} \alpha_{1} \bar{\alpha}_{1}(1-\epsilon)\left((\boldsymbol{k}+\boldsymbol{\ell}+\boldsymbol{r})^{2}(\boldsymbol{k}+\boldsymbol{r})^{2}\right. \\
& \left.+\frac{1}{\alpha_{1}^{2}} \bar{\alpha}_{1}\left((\boldsymbol{k}+\boldsymbol{r})^{2}+\alpha_{1} Q^{2}\right)\left(2 \alpha_{1}(\boldsymbol{k}+\boldsymbol{r})(\boldsymbol{k}+\boldsymbol{\ell}+\boldsymbol{r})+\bar{\alpha}_{1}\left((\boldsymbol{k}+\boldsymbol{r})^{2}+\alpha_{1} Q^{2}\right)\right)\right) \\
& +\frac{2(\boldsymbol{k}+\boldsymbol{r})^{2}}{\alpha_{1}^{2}} \mathcal{A A}_{11}^{L} \\
& \mathcal{A A}_{12}^{T}=-\frac{1}{N_{c}} \frac{2}{D_{1} D_{2}^{2} D_{4}}\left[\bar{\alpha}_{1}(\boldsymbol{k}+\boldsymbol{r})^{2}\left(\boldsymbol{k} \boldsymbol{r}+\alpha_{2} \boldsymbol{r}^{2}\right)+\alpha_{1} \alpha_{2} \bar{\alpha}_{1} \boldsymbol{r}(\boldsymbol{k}+\boldsymbol{r}) Q^{2}\right. \\
& +(1-\epsilon)\left(\left(-\alpha_{1} \bar{\alpha}_{1} \boldsymbol{k}(\boldsymbol{k}+\boldsymbol{\ell}+\boldsymbol{r})-\bar{\alpha}_{1}^{2} \boldsymbol{k}(\boldsymbol{k}+\boldsymbol{r})+\alpha_{1} \alpha_{2}(\boldsymbol{k}+\boldsymbol{\ell})(\boldsymbol{k}+\boldsymbol{\ell}+\boldsymbol{r})\right.\right. \\
& \left.+\alpha_{2} \bar{\alpha}_{1}(\boldsymbol{k}+\boldsymbol{\ell})(\boldsymbol{k}+\boldsymbol{r})\right)(\boldsymbol{k}+\boldsymbol{r})^{2} \\
& \left.\left.-\alpha_{1} \bar{\alpha}_{1}\left(\bar{\alpha}_{1} \boldsymbol{k}(\boldsymbol{k}+\boldsymbol{r})-\alpha_{2}(\boldsymbol{k}+\boldsymbol{\ell})(\boldsymbol{k}+\boldsymbol{r})\right) Q^{2}\right)\right]+\frac{2(\boldsymbol{k}+\boldsymbol{r})^{2}}{\alpha_{1}^{2}} \mathcal{A A}_{12}^{L} \\
& \mathcal{A}_{\mathcal{A}_{13}^{T}}^{T}=C_{F} \frac{4}{\alpha_{1}^{2} \alpha_{\ell} D_{1}^{2} D_{2} D_{3}} \bar{\alpha}_{1}\left[-\alpha_{1}{ }^{3}\left(\bar{\alpha}_{2} \alpha_{\ell}(\boldsymbol{k}+\boldsymbol{\ell}+\boldsymbol{r})^{2}-\bar{\alpha}_{1}^{2} \boldsymbol{\ell}^{2}\right) Q^{2}\right. \\
& +\alpha_{1}^{2}(\boldsymbol{k}+\boldsymbol{r})(\boldsymbol{k}+\boldsymbol{\ell}+\boldsymbol{r})\left(\alpha_{\ell}(\boldsymbol{k}+\boldsymbol{\ell}+\boldsymbol{r})^{2}+\bar{\alpha}_{1} \ell^{2}-\bar{\alpha}_{1} \alpha_{\ell} Q^{2}\right)+\alpha_{\ell}(\boldsymbol{k}+\boldsymbol{r})^{2} \\
& \left(-\alpha_{1} \bar{\alpha}_{2}(\boldsymbol{k}+\boldsymbol{\ell}+\boldsymbol{r})^{2}+\bar{\alpha}_{1}^{2}(\boldsymbol{k}+\boldsymbol{r})^{2}+\alpha_{1} \bar{\alpha}_{1}(\boldsymbol{k}+\boldsymbol{r})(\boldsymbol{k}+\boldsymbol{\ell}+\boldsymbol{r})-\right.
\end{aligned}
$$




$$
\begin{aligned}
& \left.\alpha_{1} \bar{\alpha}_{1}^{2}\left(-\frac{\ell^{2}}{\alpha_{\ell}}-\alpha_{1} Q^{2}\right)\right) \\
& +\alpha_{1}(1-\epsilon)\left(-\alpha_{1}{ }^{2} \bar{\alpha}_{1}^{2} \ell^{2} Q^{2}-\alpha_{1} \alpha_{\ell}(\boldsymbol{k}+\boldsymbol{r})(\boldsymbol{k}+\boldsymbol{\ell}+\boldsymbol{r})\left(\alpha_{2}(\boldsymbol{k}+\boldsymbol{\ell}+\boldsymbol{r})^{2}\right.\right. \\
& \left.-4 \bar{\alpha}_{1}(\boldsymbol{k}+\boldsymbol{r})(\boldsymbol{k}+\boldsymbol{\ell}+\boldsymbol{r})+\frac{\alpha_{1} \bar{\alpha}_{1} \ell^{2}}{\alpha_{\ell}}-\bar{\alpha}_{1}^{2} Q^{2}\right) \\
& +\alpha_{\ell}(\boldsymbol{k}+\boldsymbol{r})^{2}\left(\left(-2 \alpha_{1} \bar{\alpha}_{1}+\alpha_{2} \bar{\alpha}_{2}\right)(\boldsymbol{k}+\boldsymbol{\ell}+\boldsymbol{r})^{2}-\bar{\alpha}_{1}^{2}(\boldsymbol{k}+\boldsymbol{r})^{2}\right. \\
& \left.\left.\left.+\bar{\alpha}_{1}\left(-\alpha_{1}+\bar{\alpha}_{1}-\bar{\alpha}_{2}\right)(\boldsymbol{k}+\boldsymbol{r})(\boldsymbol{k}+\boldsymbol{\ell}+\boldsymbol{r})+\alpha_{1} \bar{\alpha}_{1}^{2}\left(-\frac{\boldsymbol{\ell}^{2}}{\alpha_{\ell}}-Q^{2}\right)\right)\right)\right] \\
& \mathcal{A} \mathcal{A}_{14}^{T}=-N_{c} \frac{1}{D_{1} D_{2}^{2} D_{5}}\left[\bar{\alpha}_{1}^{2}(\boldsymbol{k}+\boldsymbol{r})^{2}\left(-(\boldsymbol{k}+\boldsymbol{r})^{2}+\frac{\alpha_{1} \ell^{2}}{\alpha_{\ell}}-\alpha_{1} Q^{2}\right)\right. \\
& +\frac{2 \alpha_{\ell}}{\alpha_{1}}(1-\epsilon)\left(\alpha_{1} \bar{\alpha}_{1}\left((\boldsymbol{k}+\boldsymbol{r})^{2}+\alpha_{1} Q^{2}\right)\left(2(\boldsymbol{k}+\boldsymbol{\ell})(\boldsymbol{k}+\boldsymbol{r})+\boldsymbol{r}(\boldsymbol{k}+\boldsymbol{r})+\bar{\alpha}_{1} Q^{2}\right)\right. \\
& \left.+(\boldsymbol{k}+\boldsymbol{r})^{2}\left(\alpha_{1}^{2}(\boldsymbol{k}+\boldsymbol{\ell})(\boldsymbol{k}+\boldsymbol{\ell}+\boldsymbol{r})+\bar{\alpha}_{1}^{2}(\boldsymbol{k}+\boldsymbol{r})^{2}+\alpha_{1} \bar{\alpha}_{1}^{2} Q^{2}\right)\right) \\
& +\alpha_{1} \bar{\alpha}_{1}(\boldsymbol{k}+\boldsymbol{r})^{2}\left(-\boldsymbol{k}(\boldsymbol{k}+\boldsymbol{\ell})+(\boldsymbol{\ell}-\boldsymbol{r})(\boldsymbol{k}+\boldsymbol{\ell}+\boldsymbol{r})-\boldsymbol{r}^{2}\right) \\
& +\bar{\alpha}_{1}\left(-(\boldsymbol{k}+\boldsymbol{r})^{2}-\alpha_{1} Q^{2}\right)\left(\alpha_{\ell}(2(\boldsymbol{k}+\boldsymbol{\ell})(\boldsymbol{k}+\boldsymbol{r})+\boldsymbol{r}(\boldsymbol{k}+\boldsymbol{r}))\right. \\
& \left.\left.+\bar{\alpha}_{1}(2 \boldsymbol{\ell}-\boldsymbol{r})(\boldsymbol{k}+\boldsymbol{r})\right)\right] \\
& +\frac{2(\boldsymbol{k}+\boldsymbol{r})^{2}}{\alpha_{1}^{2}} \mathcal{A} \mathcal{A}_{14}^{L} \\
& \mathcal{A}_{\mathcal{A}_{15}^{T}}^{T}=N_{c} \frac{2}{\alpha_{1} \alpha_{\ell} D_{1} D_{2}{ }^{2} D_{5}}\left(\alpha_{1}^{2}+\alpha_{2}^{2}\right) \bar{\alpha}_{1}^{2}(\boldsymbol{k}+\boldsymbol{r})^{2} \boldsymbol{r}^{2}
\end{aligned}
$$

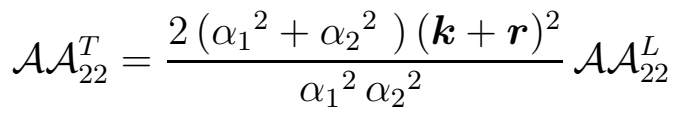

$$
\begin{aligned}
& \mathcal{A A}_{23}^{T}=-\frac{1}{N_{c}} \frac{2}{D_{1} D_{2} D_{3} D_{4}}\left[\left(-\alpha_{1}+\bar{\alpha}_{1}\right)(-\boldsymbol{k}(\boldsymbol{k}+\boldsymbol{r})(\boldsymbol{k}+\boldsymbol{\ell})(\boldsymbol{k}+\boldsymbol{\ell}+\boldsymbol{r})\right. \\
& +\boldsymbol{k}(\boldsymbol{k}+\boldsymbol{\ell})(\boldsymbol{k}+\boldsymbol{r})(\boldsymbol{k}+\boldsymbol{\ell}+\boldsymbol{r})) \\
& +\left(\alpha_{1}(\boldsymbol{k}+\boldsymbol{\ell})(\boldsymbol{k}+\boldsymbol{r})+\bar{\alpha}_{1}(\boldsymbol{k}+\boldsymbol{r})^{2}\right)\left(\frac{\left(-\alpha_{1}+\bar{\alpha}_{1}\right)}{\alpha_{1}} \boldsymbol{k}(\boldsymbol{k}+\boldsymbol{\ell}+\boldsymbol{r})\right. \\
& \left.-\alpha_{2}(\boldsymbol{k}+\boldsymbol{\ell}+\boldsymbol{r})^{2}+\frac{\alpha_{2}^{2} \bar{\alpha}_{1}}{\alpha_{1}}\left(\frac{(\boldsymbol{k}+\boldsymbol{r})^{2}}{\alpha_{1}}+\frac{\boldsymbol{\ell}^{2}}{\alpha_{\ell}}\right)\right) \\
& +\epsilon\left(( - \overline { \alpha } _ { 1 } \boldsymbol { k } ( \boldsymbol { k } + \boldsymbol { \ell } + \boldsymbol { r } ) + \alpha _ { 2 } ( \boldsymbol { k } + \boldsymbol { \ell } ) ( \boldsymbol { k } + \boldsymbol { \ell } + \boldsymbol { r } ) ) \left(\frac{\left(\alpha_{1}-\bar{\alpha}_{2}\right)}{\alpha_{1}}(\boldsymbol{k}+\boldsymbol{r})^{2}\right.\right. \\
& +2(\boldsymbol{k}+\boldsymbol{r})(\boldsymbol{k}+\boldsymbol{\ell}+\boldsymbol{r}))
\end{aligned}
$$




$$
\begin{aligned}
& +\left(-\bar{\alpha}_{1} \boldsymbol{k}(\boldsymbol{k}+\boldsymbol{r})+\alpha_{2}(\boldsymbol{k}+\boldsymbol{\ell})(\boldsymbol{k}+\boldsymbol{r})\right)\left(-\alpha_{2}(\boldsymbol{k}+\boldsymbol{\ell}+\boldsymbol{r})^{2}\right. \\
& \left.\left.\left.-2(\boldsymbol{k}+\boldsymbol{r})(\boldsymbol{k}+\boldsymbol{\ell}+\boldsymbol{r})+\alpha_{1} \bar{\alpha}_{1}\left(\frac{(\boldsymbol{k}+\boldsymbol{r})^{2}}{\alpha_{1}}+\frac{\boldsymbol{\ell}^{2}}{\alpha_{\ell}}\right)\right)\right)\right] \\
& +\frac{4(\boldsymbol{k}+\boldsymbol{r})(\boldsymbol{k}+\boldsymbol{\ell}+\boldsymbol{r})}{\alpha_{2} \bar{\alpha}_{1}} \mathcal{A A}_{23}^{L} \\
& \mathcal{A A}_{24}^{T}=N_{c} \frac{1}{D_{2}^{2} D_{4} D_{5}}\left[\frac { 2 \alpha _ { \ell } ( 1 - \epsilon ) } { \overline { \alpha } _ { 1 } } \left(\alpha_{1}\left(-\bar{\alpha}_{1} \boldsymbol{k}(\boldsymbol{k}+\boldsymbol{\ell})+\alpha_{2}(\boldsymbol{k}+\boldsymbol{\ell})^{2}\right)(\boldsymbol{k}+\boldsymbol{r})^{2}\right.\right. \\
& \left.+\bar{\alpha}_{1}\left(-\bar{\alpha}_{1} \boldsymbol{k}(\boldsymbol{k}+\boldsymbol{r})+\alpha_{2}(\boldsymbol{k}+\boldsymbol{\ell})(\boldsymbol{k}+\boldsymbol{r})\right)\left((\boldsymbol{k}+\boldsymbol{r})^{2}+\alpha_{1} Q^{2}\right)\right) \\
& +\alpha_{1}(\boldsymbol{k}+\boldsymbol{r})^{2}\left(\alpha_{\ell} \boldsymbol{k}(\boldsymbol{k}+\ell)-\alpha_{2}(\boldsymbol{k}+\boldsymbol{\ell})^{2}+\bar{\alpha}_{1} \boldsymbol{k}(\boldsymbol{\ell}-2 \boldsymbol{r})+\frac{\alpha_{2} \bar{\alpha}_{1} \ell^{2}}{\alpha_{\ell}}\right. \\
& \left.+\alpha_{2}(2 \boldsymbol{\ell}-\boldsymbol{r})(\boldsymbol{k}+\boldsymbol{\ell})\right) \\
& \left.-\alpha_{2}\left((\boldsymbol{k}+\boldsymbol{r})^{2}+\alpha_{1} Q^{2}\right)\left(\alpha_{\ell}(\boldsymbol{k}+\boldsymbol{\ell})(\boldsymbol{k}+\boldsymbol{r})+\bar{\alpha}_{1}(\boldsymbol{k}+\boldsymbol{r})^{2}+\bar{\alpha}_{1}(\boldsymbol{\ell}+\boldsymbol{r})(\boldsymbol{k}+\boldsymbol{r})\right)\right] \\
& +\frac{2(\boldsymbol{k}+\boldsymbol{r})^{2}}{\alpha_{1}^{2}} \mathcal{A}_{\mathcal{A}_{24}^{L}}^{L} \\
& \mathcal{A A}_{25}^{T}=-N_{c} \frac{2}{\alpha_{1} \alpha_{\ell} D_{2}^{2} D_{4} D_{5}} \alpha_{2}\left(\alpha_{1}^{2}+\alpha_{2}^{2}\right) \bar{\alpha}_{1}(\boldsymbol{k}+\boldsymbol{r})^{2} \boldsymbol{r}^{2} \\
& \mathcal{A A}_{33}^{T}=2\left[\frac{(\boldsymbol{k}+\boldsymbol{\ell}+\boldsymbol{r})^{2}}{\bar{\alpha}_{1}^{2}}+\frac{\boldsymbol{\ell}^{2}}{\alpha_{\ell}^{2}}\right] \mathcal{A} \mathcal{A}_{33}^{L} \\
& \mathcal{A}_{34}^{T}=-N_{c} \frac{1}{D_{1} D_{2} D_{3} D_{5}}\left[2 \epsilon \left(\frac{-2}{\alpha_{2}} \bar{\alpha}_{1} \alpha_{\ell}((\boldsymbol{k}+\boldsymbol{r})(\boldsymbol{k}+\boldsymbol{\ell}+\boldsymbol{r}))^{2}\right.\right. \\
& +(\boldsymbol{k}+\boldsymbol{\ell})(\boldsymbol{k}+\boldsymbol{r})\left(-\alpha_{2} \alpha_{\ell}(\boldsymbol{k}+\boldsymbol{\ell}+\boldsymbol{r})^{2}+\bar{\alpha}_{1} \alpha_{\ell}(\boldsymbol{k}+\boldsymbol{r})^{2}+\alpha_{1} \bar{\alpha}_{1} \boldsymbol{\ell}^{2}\right) \\
& +\alpha_{1} \bar{\alpha}_{1}^{2} \ell^{2} Q^{2}+\frac{\alpha_{\ell}}{\alpha_{1} \alpha_{2}}(\boldsymbol{k}+\boldsymbol{r})(\boldsymbol{k}+\boldsymbol{\ell}+\boldsymbol{r})\left(\bar{\alpha}_{1}\left(2 \alpha_{1} \bar{\alpha}_{2}+\alpha_{2}\left(-\bar{\alpha}_{1}+\bar{\alpha}_{2}\right)\right)(\boldsymbol{k}+\boldsymbol{r})^{2}\right. \\
& \left.+2 \alpha_{1} \alpha_{2} \boldsymbol{\ell}(\boldsymbol{k}+\boldsymbol{\ell})-\alpha_{1} \alpha_{2} \bar{\alpha}_{1}^{2} Q^{2}\right) \\
& +\bar{\alpha}_{1}(\boldsymbol{k}+\boldsymbol{r})^{2}\left(\frac{\left(\alpha_{1}-\bar{\alpha}_{2}\right) \alpha_{\ell}(\boldsymbol{k}+\boldsymbol{\ell})(\boldsymbol{k}+\boldsymbol{\ell}+\boldsymbol{r})}{\alpha_{1} \bar{\alpha}_{1}}+\alpha_{\ell}(\boldsymbol{k}+\boldsymbol{\ell}+\boldsymbol{r})^{2}\right. \\
& \left.\left.+\frac{\bar{\alpha}_{1} \alpha_{\ell}(\boldsymbol{k}+\boldsymbol{r})^{2}}{\alpha_{1}}+\bar{\alpha}_{1} \ell^{2}+\bar{\alpha}_{1} \alpha_{\ell} Q^{2}\right)\right) \\
& +\frac{3\left(\alpha_{1}-\bar{\alpha}_{1}\right) \bar{\alpha}_{1}(\boldsymbol{k}+\boldsymbol{r})^{2} \boldsymbol{r}(\boldsymbol{k}+\boldsymbol{\ell}+\boldsymbol{r})}{\alpha_{1}} \\
& +\left(\left(\alpha_{1}-\bar{\alpha}_{1}\right)(\boldsymbol{k}+\boldsymbol{\ell})(\boldsymbol{k}+\boldsymbol{r})+\frac{\bar{\alpha}_{1}^{2}(\boldsymbol{k}+\boldsymbol{r})^{2}}{\alpha_{1}}+\alpha_{1} \bar{\alpha}_{1} \bar{\alpha}_{2} Q^{2}\right)(\boldsymbol{\ell}+\boldsymbol{r})(\boldsymbol{k}+\boldsymbol{\ell}+\boldsymbol{r}) \\
& -\frac{1}{\alpha_{1}^{2} \alpha_{\ell}}\left(\alpha_{1}^{2} \alpha_{\ell}(\boldsymbol{k}+\boldsymbol{\ell}+\boldsymbol{r})^{2}-\alpha_{2} \bar{\alpha}_{1} \alpha_{\ell}(\boldsymbol{k}+\boldsymbol{r})^{2}-\alpha_{1} \alpha_{2} \bar{\alpha}_{1} \boldsymbol{\ell}^{2}\right)
\end{aligned}
$$




$$
\begin{aligned}
& \cdot\left(2 \alpha_{1} \alpha_{\ell}(\boldsymbol{k}+\boldsymbol{\ell})(\boldsymbol{k}+\boldsymbol{r})+\bar{\alpha}_{1} \alpha_{\ell}(\boldsymbol{k}+\boldsymbol{r})^{2}+\alpha_{1} \bar{\alpha}_{1}(\boldsymbol{\ell}-2 \boldsymbol{r})(\boldsymbol{k}+\boldsymbol{r})\right) \\
& -(\boldsymbol{k}+\boldsymbol{\ell})(\boldsymbol{k}+\boldsymbol{\ell}+\boldsymbol{r})\left(\alpha_{\ell}(\boldsymbol{k}+\boldsymbol{r})^{2}+\alpha_{1} \bar{\alpha}_{2} \alpha_{\ell} Q^{2}+\left(\alpha_{1}-\bar{\alpha}_{1}\right)(\boldsymbol{\ell}+\boldsymbol{r})(\boldsymbol{k}+\boldsymbol{r})\right) \\
& +(\boldsymbol{k}+\boldsymbol{r})(\boldsymbol{k}+\boldsymbol{\ell}+\boldsymbol{r})\left(\frac { \overline { \alpha } _ { 1 } } { \alpha _ { 1 } \alpha _ { 2 } } \left(\alpha_{2}^{2} \bar{\alpha}_{1}-\alpha_{1} \alpha_{2} \bar{\alpha}_{2}+2 \alpha_{2}\left(\bar{\alpha}_{1}-\bar{\alpha}_{2}\right) \alpha_{\ell}\right.\right. \\
& \left.-4 \alpha_{1} \bar{\alpha}_{2} \alpha_{\ell}\right)(\boldsymbol{k}+\boldsymbol{r})^{2}-2 \bar{\alpha}_{1} \bar{\alpha}_{2} \alpha_{\ell} Q^{2} \\
& -\frac{\left(-\alpha_{1}+\bar{\alpha}_{1}\right)}{\alpha_{\ell}}\left(-\alpha_{\ell}(\boldsymbol{k}+\boldsymbol{\ell})^{2}+\bar{\alpha}_{1} \ell^{2}-\bar{\alpha}_{1} \alpha_{\ell} Q^{2}\right) \\
& +\frac{\left(-\alpha_{1}+\bar{\alpha}_{1}\right)}{\alpha_{2}}\left(2 \alpha_{\ell}(\boldsymbol{k}+\boldsymbol{\ell})(\boldsymbol{k}+\boldsymbol{r})-\alpha_{2}(\boldsymbol{\ell}+\boldsymbol{r})(\boldsymbol{k}+\boldsymbol{\ell})\right. \\
& \left.\left.\left.-2 \bar{\alpha}_{1}(\boldsymbol{\ell}+\boldsymbol{r})(\boldsymbol{k}+\boldsymbol{r})\right)\right)\right]+\frac{4(\boldsymbol{k}+\boldsymbol{r})(\boldsymbol{k}+\boldsymbol{\ell}+\boldsymbol{r})}{\alpha_{2} \bar{\alpha}_{1}} \mathcal{A}_{34}^{L} \\
& \mathcal{A A}_{35}^{T}=-N_{c} \frac{2}{\alpha_{\ell} D_{1} D_{2} D_{3} D_{5}} \bar{\alpha}_{1}\left(\alpha_{2} \bar{\alpha}_{1}+\alpha_{1} \bar{\alpha}_{2}\right)(\boldsymbol{k}+\boldsymbol{r})(\boldsymbol{k}+\boldsymbol{\ell}+\boldsymbol{r}) \boldsymbol{r}^{2} \\
& \mathcal{A A}_{44}^{T}=N_{c} \frac{2}{D_{2}{ }^{2} D_{5}{ }^{2}}\left[\frac { 2 \alpha _ { \ell } { } ^ { 2 } } { \alpha _ { 1 } \overline { \alpha } _ { 1 } } ( 1 - \epsilon ) \left(\alpha_{1}{ }^{2}(\boldsymbol{k}+\boldsymbol{\ell})^{2}(\boldsymbol{k}+\boldsymbol{r})^{2}+\bar{\alpha}_{1}^{2}(\boldsymbol{k}+\boldsymbol{r})^{4}\right.\right. \\
& +\alpha_{1}^{2} \bar{\alpha}_{1}(\boldsymbol{k}+\boldsymbol{\ell})(\boldsymbol{k}+\boldsymbol{r}) Q^{2} \\
& \left.+\alpha_{1} \bar{\alpha}_{1}\left(2(\boldsymbol{k}+\boldsymbol{r})^{2}+\alpha_{1} Q^{2}\right)\left((\boldsymbol{k}+\boldsymbol{\ell})(\boldsymbol{k}+\boldsymbol{r})+\bar{\alpha}_{1} Q^{2}\right)\right) \\
& +\alpha_{1}\left(-\alpha_{\ell}(\boldsymbol{k}+\boldsymbol{\ell})^{2}(\boldsymbol{k}+\boldsymbol{r})^{2}+2 \bar{\alpha}_{1}(\boldsymbol{k}+\boldsymbol{r})^{2}\left((\boldsymbol{\ell}-\boldsymbol{r})^{2}+\boldsymbol{r}^{2}\right)\right. \\
& \left.+\alpha_{\ell}(\boldsymbol{k}+\boldsymbol{r})^{2}\left(-\frac{\bar{\alpha}_{1}(\boldsymbol{k}+\boldsymbol{r})^{2}}{\alpha_{1}}-\bar{\alpha}_{1} Q^{2}+3(\boldsymbol{\ell}-\boldsymbol{r})(\boldsymbol{k}+\boldsymbol{\ell})\right)\right) \\
& \left.-3 \alpha_{\ell}\left((\boldsymbol{k}+\boldsymbol{r})^{2}+\alpha_{1} Q^{2}\right)\left(\alpha_{\ell}(\boldsymbol{k}+\boldsymbol{\ell})(\boldsymbol{k}+\boldsymbol{r})+\bar{\alpha}_{1}(\boldsymbol{\ell}-\boldsymbol{r})(\boldsymbol{k}+\boldsymbol{r})\right)\right] \\
& +\frac{2(\boldsymbol{k}+\boldsymbol{r})^{2}}{\alpha_{1}^{2}} \mathcal{A A}_{44}^{L} \\
& \mathcal{A A}_{45}^{T}=-N_{c} \frac{2}{\alpha_{1} D_{2}{ }^{2} D_{5}{ }^{2}}\left(\alpha_{1}^{2}+\alpha_{2}^{2}\right) \bar{\alpha}_{1}(\boldsymbol{k}+\boldsymbol{r})^{2} \boldsymbol{r}^{2} \\
& \mathcal{A} \mathcal{A}_{55}^{T}=0 \\
& \mathcal{A B}_{11}^{T}=-\frac{1}{N_{c}} \frac{2}{D_{1} D_{2} D_{6} D_{7}}\left[\left(\bar{\alpha}_{1} \boldsymbol{k}(\boldsymbol{k}+\boldsymbol{r})+\alpha_{1}(\boldsymbol{k}+\boldsymbol{r})(\boldsymbol{k}+\boldsymbol{\ell}+\boldsymbol{r})\right)\right. \\
& \cdot\left((\boldsymbol{k}+\boldsymbol{\ell})^{2}-2 \bar{\alpha}_{1}(\boldsymbol{k}+\boldsymbol{\ell})(\boldsymbol{k}+\boldsymbol{r})+\alpha_{2} \bar{\alpha}_{1} Q^{2}\right) \\
& +\left(\bar{\alpha}_{1} \boldsymbol{k}(\boldsymbol{k}+\boldsymbol{\ell})+\alpha_{1}(\boldsymbol{k}+\boldsymbol{\ell})(\boldsymbol{k}+\boldsymbol{\ell}+\boldsymbol{r})\right)\left(-2 \alpha_{1}(\boldsymbol{k}+\boldsymbol{\ell})(\boldsymbol{k}+\boldsymbol{r})\right. \\
& \left.+(\boldsymbol{k}+\boldsymbol{r})^{2}+\alpha_{1} \bar{\alpha}_{2} Q^{2}\right)
\end{aligned}
$$




$$
\begin{aligned}
& +\epsilon\left(( \boldsymbol { k } + \boldsymbol { \ell } ) ( \boldsymbol { k } + \boldsymbol { r } ) \left(\left(\alpha_{1} \bar{\alpha}_{1}+\alpha_{2} \bar{\alpha}_{2}\right) \boldsymbol{k}(\boldsymbol{k}+\boldsymbol{\ell}+\boldsymbol{r})-2 \bar{\alpha}_{1} \bar{\alpha}_{2} \boldsymbol{k}(\boldsymbol{k}+\boldsymbol{r})\right.\right. \\
& \left.-2 \alpha_{1} \alpha_{2}(\boldsymbol{k}+\boldsymbol{\ell})(\boldsymbol{k}+\boldsymbol{\ell}+\boldsymbol{r})+2 \alpha_{1} \bar{\alpha}_{1}(\boldsymbol{k}+\boldsymbol{\ell})(\boldsymbol{k}+\boldsymbol{r})\right) \\
& +\left(-\alpha_{1} \bar{\alpha}_{1}+\alpha_{2} \bar{\alpha}_{2}\right)(\boldsymbol{k}(\boldsymbol{k}+\boldsymbol{r})(\boldsymbol{k}+\boldsymbol{\ell})(\boldsymbol{k}+\boldsymbol{\ell}+\boldsymbol{r}) \\
& -\boldsymbol{k}(\boldsymbol{k}+\boldsymbol{\ell})(\boldsymbol{k}+\boldsymbol{r})(\boldsymbol{k}+\boldsymbol{\ell}+\boldsymbol{r})) \\
& +\bar{\alpha}_{1}(\boldsymbol{k}+\boldsymbol{r})^{2}\left(\boldsymbol{k}(\boldsymbol{k}+\boldsymbol{\ell})+\alpha_{1} \bar{\alpha}_{1} Q^{2}\right) \\
& +\alpha_{1}(\boldsymbol{k}+\boldsymbol{\ell})^{2}\left((\boldsymbol{k}+\boldsymbol{r})(\boldsymbol{k}+\boldsymbol{\ell}+\boldsymbol{r})+\alpha_{1} \bar{\alpha}_{1} Q^{2}\right) \\
& \left.\left.+\alpha_{1} \bar{\alpha}_{1} Q^{2}\left(\bar{\alpha}_{1} \boldsymbol{k}(\boldsymbol{k}+\boldsymbol{\ell})+\alpha_{1}(\boldsymbol{k}+\boldsymbol{r})(\boldsymbol{k}+\boldsymbol{\ell}+\boldsymbol{r})+\alpha_{1} \bar{\alpha}_{1} Q^{2}\right)\right)\right] \\
& \mathcal{A B}_{12}^{T}=-C_{F} \frac{4}{D_{1} D_{2} D_{3} D_{7}}\left[\frac{1}{\alpha_{1}}\left(\alpha_{2} \bar{\alpha}_{1}^{2}-2 \alpha_{1} \bar{\alpha}_{1} \bar{\alpha}_{2}-\alpha_{1} \bar{\alpha}_{2}^{2}\right)(\boldsymbol{k}+\boldsymbol{\ell})(\boldsymbol{k}+\boldsymbol{\ell}+\boldsymbol{r})(\boldsymbol{k}+\boldsymbol{r})^{2}\right. \\
& -\alpha_{1} \bar{\alpha}_{2}^{2}(\boldsymbol{k}+\boldsymbol{\ell})(\boldsymbol{k}+\boldsymbol{\ell}+\boldsymbol{r}) Q^{2} \\
& +\frac{1}{\alpha_{1}}(\boldsymbol{k}+\boldsymbol{\ell})(\boldsymbol{k}+\boldsymbol{r})\left(\alpha_{1}\left(\alpha_{2} \bar{\alpha}_{1}+\alpha_{1} \bar{\alpha}_{2}\right)(\boldsymbol{k}+\boldsymbol{\ell}+\boldsymbol{r})^{2}\right. \\
& \left.+\bar{\alpha}_{1}\left(\bar{\alpha}_{1}-\bar{\alpha}_{2}\right) \bar{\alpha}_{2}(\boldsymbol{k}+\boldsymbol{r})^{2}+\alpha_{1} \bar{\alpha}_{1} \bar{\alpha}_{2}\left(4(\boldsymbol{k}+\boldsymbol{r})(\boldsymbol{k}+\boldsymbol{\ell}+\boldsymbol{r})-\bar{\alpha}_{2} Q^{2}\right)\right) \\
& +\epsilon\left(\frac{\left(\alpha_{1} \bar{\alpha}_{1}^{2}+2 \alpha_{1} \bar{\alpha}_{1} \bar{\alpha}_{2}-\alpha_{2} \bar{\alpha}_{2}^{2}\right)}{\alpha_{1}}(\boldsymbol{k}+\boldsymbol{\ell})(\boldsymbol{k}+\boldsymbol{\ell}+\boldsymbol{r})(\boldsymbol{k}+\boldsymbol{r})^{2}\right. \\
& +\alpha_{1} \bar{\alpha}_{1}^{2}(\boldsymbol{k}+\boldsymbol{\ell})(\boldsymbol{k}+\boldsymbol{\ell}+\boldsymbol{r}) Q^{2} \\
& +\frac{1}{\alpha_{1}}(\boldsymbol{k}+\boldsymbol{\ell})(\boldsymbol{k}+\boldsymbol{r})\left(\alpha_{1}\left(\alpha_{1} \bar{\alpha}_{1}+\alpha_{2} \bar{\alpha}_{2}\right)(\boldsymbol{k}+\boldsymbol{\ell}+\boldsymbol{r})^{2}\right. \\
& \left.\left.\left.-\bar{\alpha}_{1}\left(\bar{\alpha}_{1}-\bar{\alpha}_{2}\right) \bar{\alpha}_{2}(\boldsymbol{k}+\boldsymbol{r})^{2}+\alpha_{1} \bar{\alpha}_{1} \bar{\alpha}_{2}\left(-4(\boldsymbol{k}+\boldsymbol{r})(\boldsymbol{k}+\boldsymbol{\ell}+\boldsymbol{r})-\bar{\alpha}_{1} Q^{2}\right)\right)\right)\right] \\
& \mathcal{A B}_{13}^{T}=\frac{1}{N_{c}} \frac{2}{D_{1} D_{2} D_{4} D_{6}}\left[\left(\bar{\alpha}_{1} \boldsymbol{k}^{2}-\alpha_{2} \boldsymbol{k}(\boldsymbol{k}+\boldsymbol{\ell}+\boldsymbol{r})\right)\left(\alpha_{2}(\boldsymbol{k}+\boldsymbol{\ell})(\boldsymbol{k}+\boldsymbol{r})-\bar{\alpha}_{1}(\boldsymbol{k}+\boldsymbol{r})^{2}\right)\right. \\
& +\left(\alpha_{1}^{2}-\alpha_{2}^{2}\right)(\boldsymbol{k}(\boldsymbol{k}+\boldsymbol{r})(\boldsymbol{k}+\boldsymbol{\ell})(\boldsymbol{k}+\boldsymbol{\ell}+\boldsymbol{r})-\boldsymbol{k}(\boldsymbol{k}+\boldsymbol{\ell})(\boldsymbol{k}+\boldsymbol{r})(\boldsymbol{k}+\boldsymbol{\ell}+\boldsymbol{r})) \\
& -\alpha_{1}\left(-\boldsymbol{k}(\boldsymbol{k}+\boldsymbol{\ell}+\boldsymbol{r})+(\boldsymbol{k}+\boldsymbol{\ell})^{2}+\frac{\bar{\alpha}_{1}}{\alpha_{\ell}} \boldsymbol{\ell}^{2}\right) \\
& \cdot\left(\alpha_{1}(\boldsymbol{k}+\boldsymbol{\ell})(\boldsymbol{k}+\boldsymbol{r})+\bar{\alpha}_{1}(\boldsymbol{k}+\boldsymbol{r})^{2}+\alpha_{1} \bar{\alpha}_{1} Q^{2}\right) \\
& +\epsilon\left(-\bar{\alpha}_{1}\left(-\bar{\alpha}_{1} \boldsymbol{k}^{2}+\alpha_{2} \boldsymbol{k}(\boldsymbol{k}+\ell)\right)(\boldsymbol{k}+\boldsymbol{r})^{2}\right. \\
& +\left(\alpha_{1}^{2}-\alpha_{2}^{2}\right)(-\boldsymbol{k}(\boldsymbol{k}+\boldsymbol{r})(\boldsymbol{k}+\boldsymbol{\ell})(\boldsymbol{k}+\boldsymbol{\ell}+\boldsymbol{r})
\end{aligned}
$$




$$
\begin{aligned}
& +\boldsymbol{k}(\boldsymbol{k}+\boldsymbol{\ell}+\boldsymbol{r})(\boldsymbol{k}+\boldsymbol{\ell})(\boldsymbol{k}+\boldsymbol{r})-\boldsymbol{k}(\boldsymbol{k}+\ell)(\boldsymbol{k}+\boldsymbol{r})(\boldsymbol{k}+\boldsymbol{\ell}+\boldsymbol{r})) \\
& +(\boldsymbol{k}+\boldsymbol{r})(\boldsymbol{k}+\boldsymbol{\ell}+\boldsymbol{r})\left(-\alpha_{2} \bar{\alpha}_{1} \boldsymbol{k}^{2}+\alpha_{1}^{2}(\boldsymbol{k}+\boldsymbol{\ell})^{2}+\frac{\alpha_{1}^{2} \bar{\alpha}_{1}}{\alpha_{\ell}} \boldsymbol{\ell}^{2}\right) \\
& \left.\left.+\frac{\alpha_{1} \bar{\alpha}_{1}}{\alpha_{\ell}}\left(\bar{\alpha}_{1} \boldsymbol{\ell}^{2}+\alpha_{\ell} \boldsymbol{\ell}(\boldsymbol{k}+\boldsymbol{\ell})\right)\left((\boldsymbol{k}+\boldsymbol{r})^{2}+\alpha_{1} Q^{2}\right)\right)\right] \\
& +\frac{4 \boldsymbol{k}(\boldsymbol{k}+\boldsymbol{r})}{\alpha_{1}^{2}} \mathcal{A B}_{13}^{L} \\
& \mathcal{A B}_{14}^{T}=N_{c} \frac{1}{D_{1} D_{2} D_{5} D_{7}}\left[-2 \epsilon\left(\frac{-2}{\alpha_{2}} \bar{\alpha}_{1} \alpha_{\ell}[(\boldsymbol{k}+\boldsymbol{\ell})(\boldsymbol{k}+\boldsymbol{r})]^{2}\right.\right. \\
& +\frac{\left(\alpha_{1} \bar{\alpha}_{1}-\alpha_{2} \bar{\alpha}_{2}\right) \alpha_{\ell}}{\alpha_{1}}(\boldsymbol{k}+\boldsymbol{\ell})(\boldsymbol{k}+\boldsymbol{\ell}+\boldsymbol{r})(\boldsymbol{k}+\boldsymbol{r})^{2} \\
& -\alpha_{\ell}(\boldsymbol{k}+\boldsymbol{r})(\boldsymbol{k}+\boldsymbol{\ell}+\boldsymbol{r})\left((\boldsymbol{k}+\boldsymbol{\ell})^{2}+\alpha_{1} \bar{\alpha}_{1} Q^{2}\right) \\
& -\bar{\alpha}_{1} \alpha_{\ell} Q^{2}\left(\alpha_{1}(\boldsymbol{k}+\ell)^{2}+\bar{\alpha}_{1}(\boldsymbol{k}+\boldsymbol{r})^{2}+\alpha_{1} \bar{\alpha}_{1} Q^{2}\right) \\
& +\alpha_{\ell}(\boldsymbol{k}+\boldsymbol{\ell})(\boldsymbol{k}+\boldsymbol{r})(2(\boldsymbol{\ell}-\boldsymbol{r})(\boldsymbol{k}+\boldsymbol{\ell}+\boldsymbol{r}) \\
& \left.\left.+\left(\frac{2 \bar{\alpha}_{1} \bar{\alpha}_{2}}{\alpha_{2}}+\frac{\bar{\alpha}_{1}\left(-\bar{\alpha}_{1}+\bar{\alpha}_{2}\right)}{\alpha_{1}}\right)(\boldsymbol{k}+\boldsymbol{r})^{2}-\bar{\alpha}_{1}^{2} Q^{2}\right)\right) \\
& -\left((\boldsymbol{k}+\boldsymbol{r})^{2}+\alpha_{1} \bar{\alpha}_{2} Q^{2}\right)\left(-\alpha_{\ell}(\boldsymbol{k}+\boldsymbol{\ell})(\boldsymbol{k}+\boldsymbol{\ell}+\boldsymbol{r})+\bar{\alpha}_{1}(\boldsymbol{\ell}-2 \boldsymbol{r})(\boldsymbol{k}+\boldsymbol{\ell})\right) \\
& -\frac{1}{\alpha_{1}}(\boldsymbol{k}+\boldsymbol{r})^{2}\left(-\bar{\alpha}_{1} \alpha_{\ell}(\boldsymbol{k}+\boldsymbol{\ell})^{2}-\alpha_{2} \bar{\alpha}_{1}^{2} \alpha_{\ell} Q^{2}+\bar{\alpha}_{1}\left(-\alpha_{1}+\bar{\alpha}_{1}\right)(\boldsymbol{\ell}+\boldsymbol{r})(\boldsymbol{k}+\boldsymbol{\ell})\right) \\
& +(\boldsymbol{k}+\boldsymbol{\ell})(\boldsymbol{k}+\boldsymbol{r})\left[-\frac{\bar{\alpha}_{1}\left(-\alpha_{1}+\bar{\alpha}_{1}\right)\left(\alpha_{2}+2 \alpha_{\ell}\right)}{\alpha_{1} \alpha_{2}}(\boldsymbol{k}+\boldsymbol{r})^{2}\right. \\
& +\frac{2 \bar{\alpha}_{1} \bar{\alpha}_{2} \alpha_{\ell}}{\alpha_{2}}\left(\frac{1}{\alpha_{1}}(\boldsymbol{k}+\boldsymbol{r})^{2}+\alpha_{2} Q^{2}\right) \\
& +\frac{\left(-\alpha_{1}+\bar{\alpha}_{1}\right)}{\alpha_{2} \alpha_{\ell}}\left(\alpha_{2}\left(-\alpha_{\ell}(\boldsymbol{k}+\ell)^{2}+\bar{\alpha}_{1} \ell^{2}-\bar{\alpha}_{1} \alpha_{\ell} Q^{2}+\alpha_{\ell}(\boldsymbol{\ell}-2 \boldsymbol{r})(\boldsymbol{k}+\boldsymbol{\ell}+\boldsymbol{r})\right)\right. \\
& \left.\left.-2 \alpha_{\ell}\left(\alpha_{\ell}(\boldsymbol{k}+\boldsymbol{r})(\boldsymbol{k}+\boldsymbol{\ell}+\boldsymbol{r})-\bar{\alpha}_{1}(\boldsymbol{\ell}-2 \boldsymbol{r})(\boldsymbol{k}+\boldsymbol{r})\right)\right)\right] \\
& +\left(-\alpha_{1}+\bar{\alpha}_{1}\right)((\boldsymbol{k}+\boldsymbol{r})(\boldsymbol{k}+\boldsymbol{\ell}+\boldsymbol{r})(\boldsymbol{\ell}-2 \boldsymbol{r})(\boldsymbol{k}+\boldsymbol{\ell}) \\
& -(\boldsymbol{k}+\boldsymbol{\ell})(\boldsymbol{k}+\boldsymbol{\ell}+\boldsymbol{r})(\boldsymbol{\ell}-2 \boldsymbol{r})(\boldsymbol{k}+\boldsymbol{r})) \\
& \left.+\left((\boldsymbol{k}+\boldsymbol{\ell})^{2}+\alpha_{2} \bar{\alpha}_{1} Q^{2}\right)\left(2 \alpha_{\ell}(\boldsymbol{k}+\boldsymbol{r})(\boldsymbol{k}+\boldsymbol{\ell}+\boldsymbol{r})+\bar{\alpha}_{1}(\boldsymbol{\ell}+\boldsymbol{r})(\boldsymbol{k}+\boldsymbol{r})\right)\right] \\
& +\frac{4(\boldsymbol{k}+\boldsymbol{\ell})(\boldsymbol{k}+\boldsymbol{r})}{\alpha_{2} \bar{\alpha}_{1}} \mathcal{A B}_{14}^{L} \\
& \mathcal{A B}_{15}^{T}=-N_{c} \frac{2}{\alpha_{\ell} D_{1} D_{2} D_{5} D_{7}} \bar{\alpha}_{1}\left(\alpha_{2} \bar{\alpha}_{1}+\alpha_{1} \bar{\alpha}_{2}\right)(\boldsymbol{k}+\boldsymbol{\ell})(\boldsymbol{k}+\boldsymbol{r}) \boldsymbol{r}^{2}
\end{aligned}
$$




$$
\begin{aligned}
& \mathcal{A B}_{22}^{T}=-\frac{1}{N_{c}} \frac{2}{D_{2} D_{3} D_{4} D_{7}}\left[\frac{\left(\alpha_{1}-\bar{\alpha}_{1}\right)}{\alpha_{1}}(\boldsymbol{k}+\boldsymbol{r})^{2}\left(\bar{\alpha}_{2} \boldsymbol{k}(\boldsymbol{k}+\boldsymbol{\ell})+\alpha_{2}(\boldsymbol{k}+\boldsymbol{\ell})(\boldsymbol{k}+\boldsymbol{\ell}+\boldsymbol{r})\right)\right. \\
& -\frac{\left(-\alpha_{2} \bar{\alpha}_{1}+\alpha_{1} \bar{\alpha}_{2}\right)}{\bar{\alpha}_{1}}(\boldsymbol{k}+\boldsymbol{\ell})^{2}\left(\bar{\alpha}_{2} \boldsymbol{k}(\boldsymbol{k}+\boldsymbol{r})+\alpha_{2}(\boldsymbol{k}+\boldsymbol{r})(\boldsymbol{k}+\boldsymbol{\ell}+\boldsymbol{r})\right) \\
& -2(\boldsymbol{k}+\boldsymbol{\ell})(\boldsymbol{k}+\boldsymbol{r})\left(\bar{\alpha}_{2}^{2} \boldsymbol{k}(\boldsymbol{k}+\boldsymbol{r})+\alpha_{2}^{2}(\boldsymbol{k}+\boldsymbol{\ell})(\boldsymbol{k}+\boldsymbol{\ell}+\boldsymbol{r})\right. \\
& \left.+\alpha_{2} \bar{\alpha}_{2}(\boldsymbol{k}(\boldsymbol{k}+\boldsymbol{\ell})+(\boldsymbol{k}+\boldsymbol{r})(\boldsymbol{k}+\boldsymbol{\ell}+\boldsymbol{r}))\right) \\
& +\epsilon\left(( \boldsymbol { k } + \boldsymbol { \ell } ) ( \boldsymbol { k } + \boldsymbol { r } ) \left(\left(\alpha_{1} \bar{\alpha}_{1}+\alpha_{2} \bar{\alpha}_{2}\right) \boldsymbol{k}(\boldsymbol{k}+\boldsymbol{\ell}+\boldsymbol{r})-2 \bar{\alpha}_{1} \bar{\alpha}_{2} \boldsymbol{k}(\boldsymbol{k}+\boldsymbol{r})\right.\right. \\
& \left.-2 \alpha_{1} \alpha_{2}(\boldsymbol{k}+\boldsymbol{\ell})(\boldsymbol{k}+\boldsymbol{\ell}+\boldsymbol{r})\right)+2 \alpha_{2} \bar{\alpha}_{2}[(\boldsymbol{k}+\boldsymbol{\ell})(\boldsymbol{k}+\boldsymbol{r})]^{2} \\
& +\left(\alpha_{1} \bar{\alpha}_{1}-\alpha_{2} \bar{\alpha}_{2}\right)(\boldsymbol{k}(\boldsymbol{k}+\boldsymbol{r})(\boldsymbol{k}+\boldsymbol{\ell})(\boldsymbol{k}+\boldsymbol{\ell}+\boldsymbol{r})-\boldsymbol{k}(\boldsymbol{k}+\boldsymbol{\ell})(\boldsymbol{k}+\boldsymbol{r})(\boldsymbol{k}+\boldsymbol{\ell}+\boldsymbol{r})) \\
& +\left(\alpha_{1} \bar{\alpha}_{1}-\alpha_{2} \bar{\alpha}_{2}\right)\left(\frac{\bar{\alpha}_{2}}{\alpha_{1}} \boldsymbol{k}(\boldsymbol{k}+\boldsymbol{\ell})(\boldsymbol{k}+\boldsymbol{r})^{2}-\frac{\alpha_{2} \bar{\alpha}_{2}}{\alpha_{1} \bar{\alpha}_{1}}(\boldsymbol{k}+\boldsymbol{\ell})^{2}(\boldsymbol{k}+\boldsymbol{r})^{2}\right. \\
& \left.\left.\left.+\frac{\alpha_{2}}{\bar{\alpha}_{1}}(\boldsymbol{k}+\boldsymbol{\ell})^{2}(\boldsymbol{k}+\boldsymbol{r})(\boldsymbol{k}+\boldsymbol{\ell}+\boldsymbol{r})\right)\right)\right] \\
& \mathcal{A B}_{23}^{T}=-C_{F} \frac{4}{\bar{\alpha}_{1} D_{2} D_{4}^{2} D_{6}} \alpha_{1}^{2}(1-\epsilon)\left(\bar{\alpha}_{1} \boldsymbol{k}^{2}-\alpha_{2}(\boldsymbol{k}+\boldsymbol{\ell})^{2}-\frac{\alpha_{2} \bar{\alpha}_{1} \ell^{2}}{\alpha_{\ell}}\right) \boldsymbol{\ell}(\boldsymbol{k}+\boldsymbol{r}) \\
& +\frac{2 \boldsymbol{k}(\boldsymbol{k}+\boldsymbol{r})}{\alpha_{1}^{2}} \mathcal{A B}_{23}^{L} \\
& \mathcal{A B}_{24}^{T}=-N_{c} \frac{1}{D_{2} D_{4} D_{5} D_{7}}\left[2 \epsilon \left[\frac{\alpha_{2}\left(-\alpha_{1} \bar{\alpha}_{1}+\alpha_{2} \bar{\alpha}_{2}\right) \alpha_{\ell}}{\alpha_{1} \bar{\alpha}_{1}}(\boldsymbol{k}+\boldsymbol{\ell})^{2}(\boldsymbol{k}+\boldsymbol{r})^{2}\right.\right. \\
& +\alpha_{\ell} \boldsymbol{k}(\boldsymbol{k}+\boldsymbol{\ell})\left(2(\boldsymbol{k}+\boldsymbol{\ell})(\boldsymbol{k}+\boldsymbol{r})+\frac{\left(\alpha_{1} \bar{\alpha}_{1}-\alpha_{2} \bar{\alpha}_{2}\right)}{\alpha_{1}}(\boldsymbol{k}+\boldsymbol{r})^{2}\right) \\
& -\alpha_{\ell} \boldsymbol{k}(\boldsymbol{k}+\boldsymbol{r})\left((\boldsymbol{k}+\boldsymbol{\ell})^{2}+2(\boldsymbol{k}+\boldsymbol{\ell})(\boldsymbol{k}+\boldsymbol{r})+\alpha_{1} \bar{\alpha}_{1} Q^{2}\right) \\
& \left.-\frac{\alpha_{2} \alpha_{\ell}}{\bar{\alpha}_{1}}(\boldsymbol{k}+\boldsymbol{\ell})(\boldsymbol{k}+\boldsymbol{r})\left((\boldsymbol{k}+\boldsymbol{\ell})^{2}-2(\boldsymbol{k}+\boldsymbol{\ell})(\boldsymbol{k}+\boldsymbol{r})-\alpha_{1} \bar{\alpha}_{1} Q^{2}\right)\right] \\
& +(\boldsymbol{k}+\boldsymbol{\ell})(\boldsymbol{k}+\boldsymbol{r})\left(\frac{2 \alpha_{2} \alpha_{\ell}\left((\boldsymbol{k}+\boldsymbol{\ell})^{2}+\alpha_{2} \bar{\alpha}_{1} Q^{2}\right)}{\bar{\alpha}_{1}}\right. \\
& +\left(\bar{\alpha}_{1}-\alpha_{1}\right)\left(\alpha_{2}\left(-\frac{(\boldsymbol{k}+\boldsymbol{\ell})^{2}}{\bar{\alpha}_{1}}-\frac{(\boldsymbol{k}+\boldsymbol{r})^{2}}{\alpha_{1}}+\frac{\boldsymbol{\ell}^{2}}{\alpha_{\ell}}-Q^{2}\right)\right. \\
& \left.\left.+\frac{1}{\bar{\alpha}_{1}}\left(-2 \alpha_{\ell} \boldsymbol{k}(\boldsymbol{k}+\boldsymbol{\ell})+\bar{\alpha}_{1} \boldsymbol{k}(\boldsymbol{\ell}-2 \boldsymbol{r})+2 \alpha_{2}(\boldsymbol{\ell}-2 \boldsymbol{r})(\boldsymbol{k}+\boldsymbol{\ell})\right)\right)\right) \\
& +\left(\bar{\alpha}_{1}-\alpha_{1}\right)(-\boldsymbol{k}(\boldsymbol{k}+\boldsymbol{r})(\boldsymbol{\ell}-2 \boldsymbol{r})(\boldsymbol{k}+\boldsymbol{\ell})+\boldsymbol{k}(\boldsymbol{k}+\boldsymbol{\ell})(\boldsymbol{\ell}-2 \boldsymbol{r})(\boldsymbol{k}+\boldsymbol{r}) \\
& -\frac{1}{\alpha_{1}}(\boldsymbol{k}+\boldsymbol{r})^{2}\left(2 \alpha_{\ell} \boldsymbol{k}(\boldsymbol{k}+\boldsymbol{\ell})+\alpha_{2}(\boldsymbol{\ell}+\boldsymbol{r})(\boldsymbol{k}+\boldsymbol{\ell})\right)
\end{aligned}
$$




$$
\begin{aligned}
& \left.+\frac{1}{\bar{\alpha}_{1}}(\boldsymbol{k}+\boldsymbol{\ell})^{2}\left(\alpha_{\ell} \boldsymbol{k}(\boldsymbol{k}+\boldsymbol{r})-\alpha_{2}(\boldsymbol{\ell}-2 \boldsymbol{r})(\boldsymbol{k}+\boldsymbol{r})\right)\right) \\
& \left.+\frac{\alpha_{2}}{\alpha_{1}}\left((\boldsymbol{k}+\ell)^{2}+\alpha_{2} \bar{\alpha}_{1} Q^{2}\right)\left(\alpha_{\ell}(\boldsymbol{k}+\boldsymbol{r})^{2}+\alpha_{1}(\boldsymbol{\ell}+\boldsymbol{r})(\boldsymbol{k}+\boldsymbol{r})\right)\right] \\
& +\frac{4(\boldsymbol{k}+\boldsymbol{\ell})(\boldsymbol{k}+\boldsymbol{r})}{\alpha_{2} \bar{\alpha}_{1}} \mathcal{A B}_{24}^{L} \\
& \mathcal{A B}_{25}^{T}=N_{c} \frac{2}{\alpha_{\ell} D_{2} D_{4} D_{5} D_{7}} \alpha_{2}\left(\alpha_{2} \bar{\alpha}_{1}+\alpha_{1} \bar{\alpha}_{2}\right)(\boldsymbol{k}+\boldsymbol{\ell})(\boldsymbol{k}+\boldsymbol{r}) \boldsymbol{r}^{2} \\
& \mathcal{A B}_{33}^{T}=-\frac{1}{N_{c}} \frac{2}{D_{1} D_{3} D_{4} D_{6}}\left[-\left(\alpha_{1}(\boldsymbol{k}+\boldsymbol{\ell})(\boldsymbol{k}+\boldsymbol{\ell}+\boldsymbol{r})+\bar{\alpha}_{1}(\boldsymbol{k}+\boldsymbol{r})(\boldsymbol{k}+\boldsymbol{\ell}+\boldsymbol{r})\right)\right. \\
& \cdot\left(-\bar{\alpha}_{1} \boldsymbol{k}^{2}+2 \alpha_{1} \boldsymbol{k}(\boldsymbol{k}+\boldsymbol{\ell}+\boldsymbol{r})+\alpha_{1} \bar{\alpha}_{2}\left(\frac{(\boldsymbol{k}+\boldsymbol{\ell})^{2}}{\bar{\alpha}_{1}}+\frac{\boldsymbol{\ell}^{2}}{\alpha_{\ell}}\right)\right) \\
& -\left(\alpha_{1} \boldsymbol{k}(\boldsymbol{k}+\boldsymbol{\ell})+\bar{\alpha}_{1} \boldsymbol{k}(\boldsymbol{k}+\boldsymbol{r})\right)\left(2 \bar{\alpha}_{1} \boldsymbol{k}(\boldsymbol{k}+\boldsymbol{\ell}+\boldsymbol{r})-\alpha_{1}(\boldsymbol{k}+\boldsymbol{\ell}+\boldsymbol{r})^{2}\right. \\
& \left.+\alpha_{2} \bar{\alpha}_{1}\left(\frac{(\boldsymbol{k}+\boldsymbol{r})^{2}}{\alpha_{1}}+\frac{\ell^{2}}{\alpha_{\ell}}\right)\right) \\
& +\epsilon\left(\left(\alpha_{1} \bar{\alpha}_{1}+\alpha_{2} \bar{\alpha}_{2}\right) \boldsymbol{k}(\boldsymbol{k}+\boldsymbol{\ell}+\boldsymbol{r})(\boldsymbol{k}+\boldsymbol{\ell})(\boldsymbol{k}+\boldsymbol{r})\right. \\
& +\bar{\alpha}_{2} \bar{\alpha}_{1}\left(-2 \boldsymbol{k}(\boldsymbol{k}+\boldsymbol{\ell}+\boldsymbol{r}) \boldsymbol{k}(\boldsymbol{k}+\boldsymbol{r})+\boldsymbol{k}^{2}(\boldsymbol{k}+\boldsymbol{r})(\boldsymbol{k}+\boldsymbol{\ell}+\boldsymbol{r})\right) \\
& +\bar{\alpha}_{2} \alpha_{2}(\boldsymbol{k}(\boldsymbol{k}+\boldsymbol{r})(\boldsymbol{k}+\boldsymbol{\ell})(\boldsymbol{k}+\boldsymbol{\ell}+\boldsymbol{r})-\boldsymbol{k}(\boldsymbol{k}+\boldsymbol{\ell})(\boldsymbol{k}+\boldsymbol{r})(\boldsymbol{k}+\boldsymbol{\ell}+\boldsymbol{r})) \\
& +\alpha_{1} \alpha_{2}\left(-2 \boldsymbol{k}(\boldsymbol{k}+\boldsymbol{\ell}+\boldsymbol{r})(\boldsymbol{k}+\boldsymbol{\ell})(\boldsymbol{k}+\boldsymbol{\ell}+\boldsymbol{r})+\boldsymbol{k}(\boldsymbol{k}+\boldsymbol{\ell})(\boldsymbol{k}+\boldsymbol{\ell}+\boldsymbol{r})^{2}\right) \\
& -\alpha_{1} \bar{\alpha}_{1}(\boldsymbol{k}(\boldsymbol{k}+\boldsymbol{r})(\boldsymbol{k}+\boldsymbol{\ell})(\boldsymbol{k}+\boldsymbol{\ell}+\boldsymbol{r})-\boldsymbol{k}(\boldsymbol{k}+\boldsymbol{\ell})(\boldsymbol{k}+\boldsymbol{r})(\boldsymbol{k}+\boldsymbol{\ell}+\boldsymbol{r})) \\
& +\frac{\alpha_{1} \bar{\alpha}_{1}^{2}}{\alpha_{\ell}}\left(-\alpha_{\ell}(\boldsymbol{k}+\boldsymbol{r})(\boldsymbol{k}+\boldsymbol{\ell}+\boldsymbol{r})+\alpha_{1} \boldsymbol{\ell}^{2}\right)\left(\frac{(\boldsymbol{k}+\boldsymbol{\ell})^{2}}{\bar{\alpha}_{1}}+\frac{\boldsymbol{\ell}^{2}}{\alpha_{\ell}}\right) \\
& +\frac{\alpha_{1}^{2} \bar{\alpha}_{1}}{\alpha_{\ell}}\left(-\alpha_{\ell} \boldsymbol{k}(\boldsymbol{k}+\boldsymbol{\ell})+\bar{\alpha}_{1} \ell^{2}\right)\left(\frac{(\boldsymbol{k}+\boldsymbol{r})^{2}}{\alpha_{1}}+\frac{\ell^{2}}{\alpha_{\ell}}\right) \\
& -\alpha_{1} \bar{\alpha}_{1}\left(-2(\boldsymbol{k}(\boldsymbol{k}+\boldsymbol{\ell}+\boldsymbol{r}))^{2}+\boldsymbol{k}^{2}(\boldsymbol{k}+\boldsymbol{\ell}+\boldsymbol{r})^{2}\right. \\
& \left.\left.\left.-(\boldsymbol{k}+\boldsymbol{\ell})^{2}(\boldsymbol{k}+\boldsymbol{r})^{2}+\frac{\alpha_{1} \bar{\alpha}_{1} \ell^{4}}{\alpha_{\ell}^{2}}\right)\right)\right] \\
& \mathcal{A B}_{34}^{T}=-N_{c} \frac{1}{D_{1} D_{3} D_{5} D_{7}}\left[2 \epsilon \left(\alpha_{\ell}\left(\alpha_{1}(\boldsymbol{k}+\ell)^{2}-\bar{\alpha}_{2}(\boldsymbol{k}+\boldsymbol{\ell})(\boldsymbol{k}+\boldsymbol{r})\right)(\boldsymbol{k}+\boldsymbol{\ell}+\boldsymbol{r})^{2}\right.\right. \\
& -\frac{\left(\bar{\alpha}_{1}-\bar{\alpha}_{2}\right) \alpha_{\ell}}{\alpha_{1}}(\boldsymbol{k}+\boldsymbol{\ell})(\boldsymbol{k}+\boldsymbol{\ell}+\boldsymbol{r})(\boldsymbol{k}+\boldsymbol{r})^{2} \\
& -\alpha_{\ell}(\boldsymbol{k}+\boldsymbol{r})(\boldsymbol{k}+\boldsymbol{\ell}+\boldsymbol{r})\left((\boldsymbol{k}+\boldsymbol{\ell})^{2}+\bar{\alpha}_{1}^{2} Q^{2}\right) \\
& \left.+\bar{\alpha}_{1} \alpha_{\ell}\left(\frac{(\boldsymbol{k}+\boldsymbol{r})^{2}}{\alpha_{1}}+\frac{\boldsymbol{\ell}^{2}}{\alpha_{\ell}}\right)\left(\bar{\alpha}_{1}(\boldsymbol{k}+\boldsymbol{\ell})(\boldsymbol{k}+\boldsymbol{r})+\alpha_{1}\left((\boldsymbol{k}+\boldsymbol{\ell})^{2}+\bar{\alpha}_{1} Q^{2}\right)\right)\right)
\end{aligned}
$$




$$
\begin{aligned}
& -\bar{\alpha}_{1}\left(\frac{(\boldsymbol{k}+\boldsymbol{r})^{2}}{\alpha_{1}}+\frac{\boldsymbol{\ell}^{2}}{\alpha_{\ell}}\right)\left(\bar{\alpha}_{1} \alpha_{\ell}(\boldsymbol{k}+\boldsymbol{\ell})(\boldsymbol{k}+\boldsymbol{r})\right. \\
& \left.+2 \alpha_{1} \alpha_{\ell}\left((\boldsymbol{k}+\boldsymbol{\ell})^{2}+\bar{\alpha}_{1} Q^{2}\right)+\alpha_{1} \bar{\alpha}_{1}(\boldsymbol{\ell}-2 \boldsymbol{r})(\boldsymbol{k}+\boldsymbol{\ell})\right) \\
& +(\boldsymbol{k}+\boldsymbol{\ell}+\boldsymbol{r})^{2}\left(-2 \alpha_{1} \alpha_{\ell}(\boldsymbol{k}+\boldsymbol{\ell})^{2}+\bar{\alpha}_{2} \alpha_{\ell}(\boldsymbol{k}+\boldsymbol{\ell})(\boldsymbol{k}+\boldsymbol{r})\right. \\
& \left.+\alpha_{1} \bar{\alpha}_{2}((\boldsymbol{\ell}-2 \boldsymbol{r})(\boldsymbol{k}+\boldsymbol{\ell}))\right) \\
& +3\left(\bar{\alpha}_{1}-\bar{\alpha}_{2}\right)(-(\boldsymbol{k}+\boldsymbol{r})(\boldsymbol{k}+\boldsymbol{\ell}+\boldsymbol{r}) \boldsymbol{r}(\boldsymbol{k}+\boldsymbol{\ell})+(\boldsymbol{k}+\boldsymbol{\ell})(\boldsymbol{k}+\boldsymbol{r}) \boldsymbol{r}(\boldsymbol{k}+\boldsymbol{\ell}+\boldsymbol{r})) \\
& +\left((\boldsymbol{k}+\boldsymbol{\ell})^{2}+\bar{\alpha}_{1}^{2} Q^{2}\right)\left(\alpha_{\ell}(\boldsymbol{k}+\boldsymbol{r})(\boldsymbol{k}+\boldsymbol{\ell}+\boldsymbol{r})+\alpha_{1}(\boldsymbol{\ell}+\boldsymbol{r})(\boldsymbol{k}+\boldsymbol{\ell}+\boldsymbol{r})\right) \\
& +\frac{\left(\bar{\alpha}_{1}-\bar{\alpha}_{2}\right)}{\alpha_{1} \bar{\alpha}_{1} \alpha_{\ell}}(\boldsymbol{k}+\boldsymbol{\ell})(\boldsymbol{k}+\boldsymbol{\ell}+\boldsymbol{r})\left(2 \bar{\alpha}_{1} \alpha_{\ell}^{2}(\boldsymbol{k}+\boldsymbol{r})^{2}+\alpha_{1}^{2} \bar{\alpha}_{1} \ell^{2}\right. \\
& \left.\left.-\alpha_{1}{ }^{2} \alpha_{\ell}\left((\boldsymbol{k}+\boldsymbol{\ell})^{2}+\bar{\alpha}_{1} Q^{2}\right)-\alpha_{1} \bar{\alpha}_{1} \alpha_{\ell}((\boldsymbol{k}+\boldsymbol{\ell})(\boldsymbol{k}+\boldsymbol{r})+\boldsymbol{\ell}(\boldsymbol{k}+\boldsymbol{r}))\right)\right] \\
& +\frac{4(\boldsymbol{k}+\boldsymbol{\ell})(\boldsymbol{k}+\boldsymbol{\ell}+\boldsymbol{r})}{\bar{\alpha}_{1}^{2}} \mathcal{A B}_{34}^{L} \\
& \mathcal{A B}_{35}^{T}=N_{c} \frac{2}{\alpha_{\ell} D_{1} D_{3} D_{5} D_{7}} \alpha_{1}\left(\bar{\alpha}_{1}^{2}+\bar{\alpha}_{2}^{2}\right)(\boldsymbol{k}+\boldsymbol{\ell})(\boldsymbol{k}+\boldsymbol{\ell}+\boldsymbol{r}) \boldsymbol{r}^{2} \\
& \mathcal{A B}_{44}^{T}=-N_{c} \frac{1}{D_{2} D_{5}{ }^{2} D_{7}}\left[-4 \alpha_{\ell}^{2}((\boldsymbol{k}+\boldsymbol{\ell})(\boldsymbol{k}+\boldsymbol{r}))^{2}+\frac{\left(\alpha_{1}+\bar{\alpha}_{1}\right) \alpha_{\ell}{ }^{2}}{\alpha_{1} \bar{\alpha}_{1}}(\boldsymbol{k}+\boldsymbol{\ell})^{2}(\boldsymbol{k}+\boldsymbol{r})^{2}\right. \\
& +4 \epsilon\left(4 \alpha_{\ell}^{2}((\boldsymbol{k}+\boldsymbol{\ell})(\boldsymbol{k}+\boldsymbol{r}))^{2}-\frac{\left(\alpha_{1}-\bar{\alpha}_{2}\right) \alpha_{\ell}^{2}}{\alpha_{1} \bar{\alpha}_{1}}(\boldsymbol{k}+\boldsymbol{\ell})^{2}(\boldsymbol{k}+\boldsymbol{r})^{2}\right. \\
& +\alpha_{\ell}^{2} Q^{2}\left(\alpha_{1}(\boldsymbol{k}+\boldsymbol{\ell})^{2}+\bar{\alpha}_{1}(\boldsymbol{k}+\boldsymbol{r})^{2}+\alpha_{1} \bar{\alpha}_{1} Q^{2}\right) \\
& -\frac{\alpha_{\ell}{ }^{2}}{\alpha_{1} \bar{\alpha}_{1}}(\boldsymbol{k}+\boldsymbol{\ell})(\boldsymbol{k}+\boldsymbol{r})\left(\alpha_{1}\left(-\alpha_{1}+\alpha_{2}\right)(\boldsymbol{k}+\boldsymbol{\ell})^{2}+\bar{\alpha}_{1}\left(-\bar{\alpha}_{1}+\bar{\alpha}_{2}\right)(\boldsymbol{k}+\boldsymbol{r})^{2}\right. \\
& \left.\left.-\alpha_{1} \bar{\alpha}_{1}\left(\alpha_{1}+\bar{\alpha}_{1}\right) Q^{2}\right)\right) \\
& +\frac{\bar{\alpha}_{1} \alpha_{\ell}}{\alpha_{1}}(\boldsymbol{k}+\boldsymbol{r})^{2}\left(\alpha_{2} \alpha_{\ell} Q^{2}-3(\boldsymbol{\ell}-\boldsymbol{r})(\boldsymbol{k}+\boldsymbol{\ell})\right) \\
& +\frac{\alpha_{1} \alpha_{\ell}}{\bar{\alpha}_{1}}(\boldsymbol{k}+\boldsymbol{\ell})^{2}\left(\bar{\alpha}_{2} \alpha_{\ell} Q^{2}+3(\boldsymbol{\ell}-\boldsymbol{r})(\boldsymbol{k}+\boldsymbol{r})\right) \\
& +(\boldsymbol{k}+\boldsymbol{\ell})(\boldsymbol{k}+\boldsymbol{r})\left(\frac{-2 \alpha_{\ell}\left(\alpha_{2} \bar{\alpha}_{1}+\alpha_{1} \bar{\alpha}_{2}+2\left(\alpha_{1}-\alpha_{2}\right) \alpha_{\ell}\right)}{\bar{\alpha}_{1}}(\boldsymbol{k}+\ell)^{2}\right. \\
& -\frac{2 \alpha_{\ell}\left(\alpha_{2} \bar{\alpha}_{1}+\alpha_{1} \bar{\alpha}_{2}+2\left(\bar{\alpha}_{1}-\bar{\alpha}_{2}\right) \alpha_{\ell}\right)}{\alpha_{1}}(\boldsymbol{k}+\boldsymbol{r})^{2} \\
& +4\left(\alpha_{2}+\bar{\alpha}_{2}\right) \alpha_{\ell}^{2} Q^{2}+2\left(\alpha_{2} \bar{\alpha}_{1}+\alpha_{1} \bar{\alpha}_{2}\right)\left(-\alpha_{\ell} Q^{2}+2 \boldsymbol{r}^{2}+2(\boldsymbol{\ell}-\boldsymbol{r})^{2}\right) \\
& \left.-6\left(\alpha_{1}-\alpha_{2}\right) \alpha_{\ell}(\boldsymbol{\ell}-\boldsymbol{r})(\boldsymbol{k}+\boldsymbol{\ell})+6\left(\bar{\alpha}_{1}-\bar{\alpha}_{2}\right) \alpha_{\ell}(\boldsymbol{\ell}-\boldsymbol{r})(\boldsymbol{k}+\boldsymbol{r})\right)
\end{aligned}
$$




$$
\begin{aligned}
& \left.+3 \alpha_{2} \bar{\alpha}_{1} \alpha_{\ell} Q^{2}(\boldsymbol{\ell}-\boldsymbol{r})(\boldsymbol{k}+\boldsymbol{r})-3 \alpha_{1} \bar{\alpha}_{2} \alpha_{\ell} Q^{2}(\boldsymbol{\ell}-\boldsymbol{r})(\boldsymbol{k}+\boldsymbol{\ell})\right] \\
\mathcal{A B}_{45}^{T}= & N_{c} \frac{2}{D_{2} D_{5}^{2} D_{7}}\left(\alpha_{2} \bar{\alpha}_{1}+\alpha_{1} \bar{\alpha}_{2}\right)(\boldsymbol{k}+\boldsymbol{\ell})(\boldsymbol{k}+\boldsymbol{r}) \boldsymbol{r}^{2} \\
\mathcal{A B}_{55}^{T}= & 0
\end{aligned}
$$

At several places we have expressed the matrix elements $\mathcal{A} \mathcal{A}_{(i j)}^{T}, \mathcal{A B}_{(i j)}^{T}$ in terms of the corresponding matrix elements for the longitudinally polarized photon; we therefore list here also the expressions for longitudinal polarizations [2]:

$$
\begin{aligned}
& \mathcal{A A}_{11}^{L}=C_{F} \frac{2}{D_{1}^{2} D_{2}^{2}}(1-\epsilon) \alpha_{1} \bar{\alpha}_{1} \boldsymbol{A}_{1}^{2} \\
& \mathcal{A A}_{12}^{L}=-\frac{1}{N_{c}} \frac{1}{D_{1} D_{2}^{2} D_{4}}\left((1-\epsilon) \alpha_{1} \alpha_{2} \boldsymbol{A}_{1} \boldsymbol{A}_{2}-\alpha_{1} \alpha_{2}^{2} \bar{\alpha}_{1} \boldsymbol{r}^{2}\right) \\
& \mathcal{A}_{\mathcal{A}_{13}^{L}}^{L}=C_{F} \frac{2}{D_{1}^{2} D_{2} D_{3}}\left((1-\epsilon) \bar{\alpha}_{1}^{2} \boldsymbol{A}_{1} \boldsymbol{A}_{3}+\bar{\alpha}_{1}^{2}(\boldsymbol{k}+\boldsymbol{r})(\boldsymbol{k}+\boldsymbol{\ell}+\boldsymbol{r})\right) \\
& \mathcal{A A}_{14}^{L}=-N_{c} \frac{1}{D_{1} D_{2}^{2} D_{5}}\left(\frac{1}{2 \alpha_{\ell}} \alpha_{1}\left(\alpha_{2} \bar{\alpha}_{1}+2(1-\epsilon) \alpha_{\ell}^{2}\right) \boldsymbol{A}_{1} \boldsymbol{A}_{4}\right. \\
& \left.+\frac{1}{2} \alpha_{1} \alpha_{2} \bar{\alpha}_{1}^{2} D_{2}+\frac{1}{2 \alpha_{\ell}} \alpha_{1} \alpha_{2}^{2} \bar{\alpha}_{1} \boldsymbol{A}_{1} \boldsymbol{r}-\alpha_{1} \alpha_{2}^{2} \bar{\alpha}_{1} \boldsymbol{r}^{2}\right) \\
& \mathcal{A A}_{15}^{L}=N_{c} \frac{1}{\alpha_{\ell} D_{1} D_{2}^{2} D_{5}} \alpha_{1} \alpha_{2}^{2} \bar{\alpha}_{1}^{2} \boldsymbol{r}^{2} \\
& \mathcal{A A}_{22}^{L}=C_{F} \frac{2}{\bar{\alpha}_{1} D_{2}^{2} D_{4}^{2}}(1-\epsilon) \alpha_{1} \alpha_{2}^{2} \boldsymbol{A}_{2}^{2} \\
& \mathcal{A}_{\mathcal{A}_{23}^{L}}^{L}=-\frac{1}{N_{c}} \frac{1}{D_{1} D_{2} D_{3} D_{4}}\left((1-\epsilon) \alpha_{2} \bar{\alpha}_{1} \boldsymbol{A}_{2} \boldsymbol{A}_{3}+\alpha_{2} \bar{\alpha}_{1}\left(\boldsymbol{k}+\boldsymbol{r}-\alpha_{1} \boldsymbol{r}\right)\left(\boldsymbol{k}+\boldsymbol{\ell}+\bar{\alpha}_{1} \boldsymbol{r}\right)\right) \\
& \mathcal{A A}_{24}^{L}=N_{c} \frac{1}{D_{2}^{2} D_{4} D_{5}}\left(\frac{1}{2 \alpha_{\ell} \bar{\alpha}_{1}} \alpha_{1} \alpha_{2}\left(\alpha_{2} \bar{\alpha}_{1}+2(1-\epsilon) \alpha_{\ell}^{2}\right) \boldsymbol{A}_{2} \boldsymbol{A}_{4}\right. \\
& \left.+\frac{1}{2} \alpha_{1} \alpha_{2}^{2} \bar{\alpha}_{1} D_{2}+\frac{1}{2 \alpha_{\ell}} \alpha_{1} \alpha_{2}^{2} \bar{\alpha}_{1} \boldsymbol{A}_{2} \boldsymbol{r}+\alpha_{1} \alpha_{2}^{2} \bar{\alpha}_{1} \boldsymbol{r}^{2}\right) \\
& \mathcal{A A}_{25}^{L}=-N_{c} \frac{1}{\alpha_{\ell} D_{2}^{2} D_{4} D_{5}} \alpha_{1} \alpha_{2}^{3} \bar{\alpha}_{1} \boldsymbol{r}^{2} \\
& \mathcal{A A}_{33}^{L}=C_{F} \frac{2}{\alpha_{1} D_{1}^{2} D_{3}^{2}}(1-\epsilon) \bar{\alpha}_{1}^{3} \boldsymbol{A}_{3}^{2} \\
& \mathcal{A A}_{34}^{L}=N_{c} \frac{1}{D_{1} D_{2} D_{3} D_{5}}\left(\frac{1}{2 \alpha_{\ell}} \bar{\alpha}_{1}\left(\alpha_{1} \bar{\alpha}_{1}-\alpha_{\ell}-2(1-\epsilon) \alpha_{\ell}^{2}\right) \boldsymbol{A}_{3} \boldsymbol{A}_{4}\right. \\
& -\frac{1}{2} \alpha_{1} \bar{\alpha}_{1}^{2} \bar{\alpha}_{2} D_{2}+\alpha_{1} \alpha_{2} \bar{\alpha}_{1}^{2} D_{7}+\frac{1}{2 \alpha_{\ell}} \alpha_{1} \alpha_{2} \bar{\alpha}_{1}^{2} \boldsymbol{A}_{3} \boldsymbol{r}+\alpha_{1} \alpha_{2} \bar{\alpha}_{1}^{2} \boldsymbol{r}^{2} \\
& \left.+\frac{3}{2} \alpha_{2} \bar{\alpha}_{1} \boldsymbol{A}_{3}(\boldsymbol{k}+\boldsymbol{\ell})\right)
\end{aligned}
$$




$$
\begin{aligned}
& \mathcal{A}_{35}^{L}=-N_{c} \frac{1}{\alpha_{\ell} D_{1} D_{2} D_{3} D_{5}} \alpha_{1} \alpha_{2} \bar{\alpha}_{1}^{2} \bar{\alpha}_{2} \boldsymbol{r}^{2} \\
& \mathcal{A}_{44}^{L}=N_{c} \frac{1}{D_{2}^{2} D_{5}^{2}}\left(\frac{2}{\bar{\alpha}_{1}} \boldsymbol{A}_{4}^{2} \alpha_{1}\left((1-\epsilon) \alpha_{\ell}^{2}+\alpha_{2} \bar{\alpha}_{1}\right)+\alpha_{1} \alpha_{2} \alpha_{\ell} \bar{\alpha}_{1} D_{2}+2 \alpha_{1} \alpha_{2}^{2} \bar{\alpha}_{1} \boldsymbol{r}^{2}\right) \\
& \mathcal{A A}_{45}^{L}=-N_{c} \frac{1}{D_{2}^{2} D_{5}^{2}} \alpha_{1} \alpha_{2}^{2} \bar{\alpha}_{1} \boldsymbol{r}^{2} \\
& \mathcal{A} \mathcal{A}_{55}^{L}=0 \\
& \mathcal{A B}_{11}^{L}=\frac{1}{N_{c}} \frac{1}{D_{1} D_{2} D_{6} D_{7}}\left((1-\epsilon) \alpha_{1} \bar{\alpha}_{1} \boldsymbol{A}_{1} \boldsymbol{B}_{1}+\alpha_{1} \bar{\alpha}_{1}\left(\boldsymbol{k}+\alpha_{1} \boldsymbol{r}\right)\left(\boldsymbol{k}+\boldsymbol{\ell}+\bar{\alpha}_{2} \boldsymbol{r}\right)\right) \\
& \mathcal{A B}_{12}^{L}=-C_{F} \frac{2}{D_{1} D_{2} D_{3} D_{7}}\left((1-\epsilon) \bar{\alpha}_{1} \bar{\alpha}_{2} \boldsymbol{A}_{1} \boldsymbol{B}_{2}+\bar{\alpha}_{1} \bar{\alpha}_{2}(\boldsymbol{k}+\boldsymbol{r})(\boldsymbol{k}+\boldsymbol{\ell}+\boldsymbol{r})\right) \\
& \mathcal{A B}_{13}^{L}=\frac{1}{N_{c}} \frac{1}{D_{1} D_{2} D_{4} D_{6}}\left((1-\epsilon) \alpha_{1}^{2} \boldsymbol{A}_{1} \boldsymbol{B}_{3}-\alpha_{1}^{2} \alpha_{2} \bar{\alpha}_{1} \boldsymbol{r}^{2}\right) \\
& \mathcal{A B}_{14}^{L}=N_{c} \frac{1}{D_{1} D_{2} D_{5} D_{7}}\left(\frac{1}{2 \alpha_{\ell}} \bar{\alpha}_{1}\left(\alpha_{1} \bar{\alpha}_{1}-\alpha_{\ell}-(1-\epsilon) 2 \alpha_{\ell}^{2}\right) \boldsymbol{A}_{1} \boldsymbol{B}_{4}\right. \\
& +\alpha_{1} \bar{\alpha}_{1}^{2} \bar{\alpha}_{2} D_{2}-\frac{1}{2} \alpha_{1} \alpha_{2} \bar{\alpha}_{1}^{2} D_{7}+\frac{1}{2 \alpha_{\ell}} \alpha_{1} \alpha_{2} \bar{\alpha}_{1} \bar{\alpha}_{2} \boldsymbol{A}_{1} \boldsymbol{r}-\alpha_{1} \alpha_{2} \bar{\alpha}_{1} \bar{\alpha}_{2} \boldsymbol{r}^{2} \\
& \left.-\frac{3}{2} \bar{\alpha}_{1} \bar{\alpha}_{2} \boldsymbol{A}_{1}(\boldsymbol{k}+\boldsymbol{r})\right) \\
& \mathcal{A B}_{15}^{L}=-N_{c} \frac{1}{\alpha_{\ell} D_{1} D_{2} D_{5} D_{7}} \alpha_{1} \alpha_{2} \bar{\alpha}_{1}^{2} \bar{\alpha}_{2} \boldsymbol{r}^{2} \\
& \mathcal{A B}_{22}^{L}=\frac{1}{N_{c}} \frac{1}{D_{2} D_{3} D_{4} D_{7}}\left((1-\epsilon) \alpha_{2} \bar{\alpha}_{2} \boldsymbol{A}_{2} \boldsymbol{B}_{2}+\alpha_{2} \bar{\alpha}_{2}\left(\boldsymbol{k}+\boldsymbol{r}-\alpha_{1} \boldsymbol{r}\right)\left(\boldsymbol{k}+\boldsymbol{\ell}+\bar{\alpha}_{1} \boldsymbol{r}\right)\right) \\
& \mathcal{A B}_{23}^{L}=-C_{F} \frac{2}{\bar{\alpha}_{1} D_{2} D_{4}^{2} D_{6}}(1-\epsilon) \alpha_{1}^{2} \alpha_{2} \boldsymbol{A}_{2} \boldsymbol{B}_{3} \\
& \mathcal{A B}_{24}^{L}=-N_{c} \frac{1}{D_{2} D_{4} D_{5} D_{7}}\left(\frac{1}{2 \alpha_{\ell}} \alpha_{2}\left(\alpha_{1} \bar{\alpha}_{1}-\alpha_{\ell}-2(1-\epsilon) \alpha_{\ell}^{2}\right) \boldsymbol{A}_{2} \boldsymbol{B}_{4}\right. \\
& +\alpha_{1} \alpha_{2} \bar{\alpha}_{1} \bar{\alpha}_{2} D_{2}-\frac{1}{2} \alpha_{1} \alpha_{2}^{2} \bar{\alpha}_{1} D_{7}+\frac{1}{2 \alpha_{\ell}} \alpha_{1} \alpha_{2} \bar{\alpha}_{1} \bar{\alpha}_{2} \boldsymbol{A}_{2} \boldsymbol{r}+\alpha_{1} \alpha_{2} \bar{\alpha}_{1} \bar{\alpha}_{2} \boldsymbol{r}^{2} \\
& \left.-\frac{3}{2} \alpha_{2} \bar{\alpha}_{2} \boldsymbol{A}_{2}(\boldsymbol{k}+\boldsymbol{r})\right) \\
& \mathcal{A B}_{25}^{L}=N_{c} \frac{1}{\alpha_{\ell} D_{2} D_{4} D_{5} D_{7}} \alpha_{1} \alpha_{2}^{2} \bar{\alpha}_{1} \bar{\alpha}_{2} \boldsymbol{r}^{2} \\
& \mathcal{A B}_{33}^{L}=\frac{1}{N_{c}} \frac{1}{D_{1} D_{3} D_{4} D_{6}}\left((1-\epsilon) \alpha_{1} \bar{\alpha}_{1} \boldsymbol{A}_{3} \boldsymbol{B}_{3}+\alpha_{1} \bar{\alpha}_{1}\left(\boldsymbol{k}+\boldsymbol{r}-\alpha_{1} \boldsymbol{r}\right)\left(\boldsymbol{k}+\boldsymbol{\ell}+\bar{\alpha}_{1} \boldsymbol{r}\right)\right) \\
& \mathcal{A B}_{34}^{L}=-N_{c} \frac{1}{D_{1} D_{3} D_{5} D_{7}}\left(\frac{1}{2 \alpha_{1} \alpha_{\ell}} \bar{\alpha}_{1}^{2}\left(\alpha_{1} \bar{\alpha}_{2}+2(1-\epsilon) \alpha_{\ell}^{2}\right) \boldsymbol{A}_{3} \boldsymbol{B}_{4}\right. \\
& \left.+\frac{1}{2} \alpha_{1} \bar{\alpha}_{1}^{2} \bar{\alpha}_{2} D_{7}+\frac{1}{2 \alpha_{\ell}} \alpha_{1} \bar{\alpha}_{1}^{2} \bar{\alpha}_{2} \boldsymbol{A}_{3} \boldsymbol{r}+\alpha_{1} \bar{\alpha}_{1}^{2} \bar{\alpha}_{2} \boldsymbol{r}^{2}\right)
\end{aligned}
$$




$$
\begin{aligned}
\mathcal{A B}_{35}^{L}= & N_{c} \frac{1}{\alpha_{\ell} D_{1} D_{3} D_{5} D_{7}} \alpha_{1} \bar{\alpha}_{1}^{2} \bar{\alpha}_{2}^{2} \boldsymbol{r}^{2} \\
\mathcal{A B}_{44}^{L}= & -N_{c} \frac{1}{D_{2} D_{5}^{2} D_{7}}\left(\left(2 \alpha_{1} \bar{\alpha}_{1}+\alpha_{\ell}-2(1-\epsilon) \alpha_{\ell}^{2}\right) \boldsymbol{A}_{4} \boldsymbol{B}_{4}\right. \\
& \left.+\frac{1}{2} \alpha_{1} \alpha_{\ell} \bar{\alpha}_{1} \bar{\alpha}_{2} D_{2}+\frac{1}{2} \alpha_{1} \alpha_{2} \alpha_{\ell} \bar{\alpha}_{1} D_{7}+2 \alpha_{1} \alpha_{2} \bar{\alpha}_{1} \bar{\alpha}_{2} \boldsymbol{r}^{2}-\alpha_{\ell}^{2}(\boldsymbol{k}+\boldsymbol{\ell})(\boldsymbol{k}+\boldsymbol{r})\right) \\
\mathcal{A B}_{45}^{L}= & N_{c} \frac{1}{D_{2} D_{5}^{2} D_{7}} \alpha_{1} \alpha_{2} \bar{\alpha}_{1} \bar{\alpha}_{2} \boldsymbol{r}^{2} \\
\mathcal{A B}_{55}^{L}= & 0 .
\end{aligned}
$$

Here we have used the following abbreviations:

$$
\begin{aligned}
& \boldsymbol{A}_{1}=\alpha_{2} \boldsymbol{\ell}+\alpha_{\ell}(\boldsymbol{k}+\boldsymbol{r}) \\
& \boldsymbol{A}_{2}=\bar{\alpha}_{1} \boldsymbol{\ell}+\alpha_{\ell}(\boldsymbol{k}+\boldsymbol{\ell}) \\
& \boldsymbol{A}_{3}=\alpha_{1} \boldsymbol{\ell}-\alpha_{\ell}(\boldsymbol{k}+\boldsymbol{r}) \\
& \boldsymbol{A}_{4}=\alpha_{2}(\boldsymbol{\ell}-\boldsymbol{r})+\alpha_{\ell}(\boldsymbol{k}+\boldsymbol{r}) \\
& \boldsymbol{B}_{1}=\bar{\alpha}_{2} \boldsymbol{\ell}-\alpha_{\ell}(\boldsymbol{k}+\boldsymbol{\ell}) \\
& \boldsymbol{B}_{2}=\alpha_{1} \boldsymbol{\ell}-\alpha_{\ell}(\boldsymbol{k}+\boldsymbol{r}) \\
& \boldsymbol{B}_{3}=\bar{\alpha}_{1} \boldsymbol{\ell}+\alpha_{\ell}(\boldsymbol{k}+\boldsymbol{\ell}) \\
& \boldsymbol{B}_{4}=\bar{\alpha}_{2}(\boldsymbol{\ell}-\boldsymbol{r})-\alpha_{\ell}(\boldsymbol{k}+\boldsymbol{\ell}) .
\end{aligned}
$$

We conclude our listing of the squared matrix elements with a few remarks. First, similar to the longitudinal case, the sum of the squared matrix elements for the transverse photon is ultraviolet finite: individual terms diverge as $\boldsymbol{\ell}$ becomes large (for fixed $\boldsymbol{k}$ ), but in the sum these divergences cancel. Secondly, it is easy to show that the factorization properties in configuration space which for the longitudinal photon have been discussed in section $\mathrm{V}$ of [2] also hold for the transverse photon: this supports the validity of the photon wave function picture beyond the LO approximation. Finally, the limit of small $\alpha_{\ell}$ agrees with the LO BFKL calculation; we shall return to this question further below.

\section{SINGULAR LIMITS OF THE REAL CORRECTIONS}

In order to formulate finite expressions for the $q \bar{q} g$ contribution to the photon impact factor

we examine the divergent limits of the squared matrix element $\left|\mathcal{M}_{q \bar{q} g}\right|^{2}$ for the process $\gamma^{*}+q \rightarrow q \bar{q} g+q$. For the phase space of the $q \bar{q} g$-final state we have from (11), (12):

$$
d \phi_{q \bar{q} g} \frac{s d \beta_{r}}{2 \pi}=\frac{d \alpha}{2 \alpha\left(1-\alpha-\alpha_{\ell}\right)} \frac{d^{D-2} \boldsymbol{k}}{(2 \pi)^{D-1}} \frac{d \alpha_{\ell}}{2 \alpha_{\ell}} \frac{d^{D-2} \boldsymbol{\ell}}{(2 \pi)^{D-1}}
$$

In the following we suppress the superscript $(D-2)$ in the integration measure of the transverse momenta. The contribution of the $q \bar{q} g$ state to the impact factor reads:

$$
\int \frac{d \alpha}{2 \alpha\left(1-\alpha-\alpha_{\ell}\right)} \int \frac{d \boldsymbol{k}}{(2 \pi)^{D-1}} \int \frac{d \alpha_{\ell}}{2 \alpha_{\ell}} \int \frac{d \boldsymbol{\ell}}{(2 \pi)^{D-1}}\left|\Gamma_{\gamma^{*} \rightarrow q \bar{q} g}^{(0)}\right|^{2},
$$


where the square of the vertex function, $\left|\Gamma_{\gamma^{*} \rightarrow q \bar{q} g}^{(0)}\right|^{2}$, follows from (62) and (63).

We begin by examining the limit in which the gluon is either collinear to the quark or to the antiquark of the $q \bar{q} g$-system. In the latter case, the gluon momentum $\ell$ is collinear with the momentum $q-k-\ell$ of the antiquark, resulting in $D_{4}=0$ or

$$
\ell^{\prime}=\ell+\frac{\alpha_{\ell}}{1-\alpha} \boldsymbol{k}=0
$$

We approximate our results for $\left|\Gamma_{\gamma^{*} \rightarrow q \bar{q} g}^{(0), a}\right|^{2}$ in the region around the singularity, i.e. we calculate the residue of the pole at $D_{4}=0$. The collinear limit of QCD matrix elements is well known and can be taken from the literature [5]. As an additional check, we have calculated the divergent limits directly from our matrix elements. For both polarizations of the photon, the $g \| \bar{q}$ collinear limit of (134) can be written as

$$
\begin{aligned}
\left.\left.\left|\Gamma_{\gamma^{*} \rightarrow q \bar{q} g}^{(0)}\right|\right|^{2} d \phi_{q \bar{q} g} \frac{s d \beta_{r}}{2 \pi}\right|^{\mathrm{coll}, \bar{q}}=\left|\Gamma_{\gamma^{*} \rightarrow q \bar{q}}^{(0)}\right|^{2} \frac{d \alpha}{2 \alpha(1-\alpha)} \frac{d \boldsymbol{k}}{(2 \pi)^{3-2 \epsilon}} \\
\quad \times C_{F} P_{g q}\left(\frac{\alpha_{\ell}}{1-\alpha}, \epsilon\right) \frac{d \alpha_{\ell}}{(1-\alpha)} \frac{d \boldsymbol{\ell}}{(2 \pi)^{3-2 \epsilon} \boldsymbol{\ell}^{\prime 2}} \\
=\mathcal{I}_{2}(\alpha, \boldsymbol{k}) d \alpha d \boldsymbol{k} C_{F} P_{g q}\left(\frac{\alpha_{\ell}}{1-\alpha}, \epsilon\right) \frac{d \alpha_{\ell}}{(1-\alpha)} \frac{d \boldsymbol{\ell}}{(2 \pi)^{3-2 \epsilon} \boldsymbol{\ell}^{\prime 2}}
\end{aligned}
$$

where $\mathcal{I}_{2}(\alpha, \boldsymbol{k}) \equiv \mathcal{I}_{2}(\alpha, \boldsymbol{k} ; \boldsymbol{r}, Q)$ was defined in (27), (28), (29), and $C_{F} P_{g q}(z, \epsilon)$ denotes the $q \rightarrow g$ Altarelli-Parisi splitting function in $D=4-2 \epsilon$ dimensions:

$$
P_{g q}(z, \epsilon)=\frac{1+(1-z)^{2}}{z}-\epsilon z
$$

The color coefficient $C_{F}$ indicates that, for the examination of singular limits it will be convenient to use the color basis $C_{F}$ and $C_{A}$ rather than the color factors $N_{c}, C_{F}$, and $1 / N_{c}$ which appear in the matrix elements of the previous section. In this basis, the $C_{A}$ part is free from collinear singularities.

In the case where the gluon is collinear to the quark, the infrared limit is obtained from (137) by substituting $\alpha$ by $\bar{\alpha}=1-\alpha-\alpha_{\ell}$ and $\boldsymbol{k}$ by $\overline{\boldsymbol{k}}=-\boldsymbol{k}-\boldsymbol{r}-\boldsymbol{\ell}$. The "soft-collinear" limit of (137) that is needed later is obtained by retaining from the splitting function only the pole $2 / z$ :

$$
\left.\left|\Gamma_{\gamma^{*} \rightarrow q \bar{q} g}^{(0)}\right|^{2} d \phi_{q \bar{q} g} \frac{s d \beta_{r}}{2 \pi}\right|^{\text {soft, coll, } \bar{q}}=\mathcal{I}_{2}(\alpha, \boldsymbol{k}) d \alpha d \boldsymbol{k} 2 C_{F} \frac{d \alpha_{\ell}}{\alpha_{\ell}} \frac{d \boldsymbol{\ell}}{(2 \pi)^{3-2 \epsilon} \boldsymbol{\ell}^{\prime 2}} .
$$

Next, we examine the 'soft limit' where the gluon momentum $\alpha_{\ell} \rightarrow 0$ and $\ell \rightarrow 0$. Formally we substitute $\ell \rightarrow \rho \ell$ or $\ell \rightarrow \rho \ell, \alpha_{\ell} \rightarrow \rho \alpha_{\ell}$, expand around $\rho=0$ and keep only the leading term. As in the collinear case, the result is proportional to the Born approximation,

\footnotetext{
${ }^{1}$ This is nothing but the $q \leftrightarrow \bar{q}$ variables exchange at fixed gluon momentum.
} 
for both polarizations of the photon. We separate the result according to different colour structures:

$$
\left.\left|\Gamma_{\gamma^{*} \rightarrow q \bar{q} g}^{(0)}\right|^{2}\right|^{\text {soft }}=\left.C_{A}\left|\Gamma_{\gamma^{*} \rightarrow q \bar{q} g}^{(0)}\right|^{2}\right|_{C_{A}} ^{\text {soft }}+\left.C_{F}\left|\Gamma_{\gamma^{*} \rightarrow q \bar{q} g}^{(0)}\right|^{2}\right|_{C_{F}} ^{\text {soft }}
$$

with

$$
\left.\left|\Gamma_{\gamma^{*} \rightarrow q \bar{q} g}^{(0)}\right|^{2}\right|_{C_{A}} ^{\text {soft }}=\left|\Gamma_{\gamma^{*} \rightarrow q \bar{q}}^{(0)}\right|^{2} \frac{4\left(\boldsymbol{\ell}-\alpha_{\ell} \boldsymbol{a}\right) \cdot\left(\boldsymbol{\ell}+\alpha_{\ell} \boldsymbol{b}\right)}{\left(\boldsymbol{\ell}-\alpha_{\ell} \boldsymbol{a}\right)^{2}\left(\boldsymbol{\ell}+\alpha_{\ell} \boldsymbol{b}\right)^{2}}
$$

and

$$
\left.\left|\Gamma_{\gamma^{*} \rightarrow q \bar{q} g}^{(0)}\right|^{2}\right|_{C_{F}} ^{\text {soft }}=\left|\Gamma_{\gamma^{*} \rightarrow q \bar{q}}^{(0)}\right|^{2} \frac{4 \alpha_{\ell}^{2}(\boldsymbol{a}+\boldsymbol{b})^{2}}{\left(\boldsymbol{\ell}-\alpha_{\ell} \boldsymbol{a}\right)^{2}\left(\boldsymbol{\ell}+\alpha_{\ell} \boldsymbol{b}\right)^{2}},
$$

where, for convenience, we have defined

$$
\boldsymbol{a}=\frac{\boldsymbol{k}+\boldsymbol{r}}{\alpha}, \quad \boldsymbol{b}=\frac{\boldsymbol{k}}{1-\alpha} .
$$

In the soft limit, the phase space measure (133) factorizes in the product of the $q \bar{q}$ measure (25) and differentials of the gluon variables

$$
d \phi_{q \bar{q} g} \frac{s d \beta_{r}}{2 \pi} \rightarrow\left(d \phi_{q \bar{q}} \frac{s d \beta_{r}}{2 \pi}\right)\left(\frac{d \alpha_{\ell}}{2 \alpha_{\ell}} \frac{d \boldsymbol{\ell}}{(2 \pi)^{D-1}}\right) .
$$

Our result for the soft limit agrees with [⿴囗十]. We also note that, in the limit $\ell^{\prime}=\boldsymbol{\ell}+\alpha_{\ell} \boldsymbol{b} \rightarrow 0$, we reproduce from (142) and (144) the "soft-collinear" limit (139).

Finally, we have to consider the limit in which the gluon is emitted in the central rapidity region. Formally, this corresponds to the singular limit $\alpha_{\ell} \rightarrow 0$ : the matrix elements listed in the previous section are finite for $\alpha_{\ell} \rightarrow 0$, but the phase space element (12) introduces a logarithmic singularity. Consequently, when doing the subsequent $\alpha_{\ell}$ integration this divergence $\sim 1 / \alpha_{\ell}$ will produce the leading-ln $s$ logarithm. As discussed in section $\mathbb{I}$, this part of the gluon phase space belongs to final states with two large rapidity gaps and has to be subtracted from our result. This will be the topic of section VI. For the moment we only state that in the limit $\alpha_{\ell} \rightarrow 0$, for both polarizations of the photon, the sums of our matrix elements split into the product of the Born approximation and the BFKL kernel:

$$
\left.\left|\Gamma_{\gamma^{*} \rightarrow q \bar{q} g}^{(0)}\right|^{2}\right|_{\mathrm{LL}}=\left.\left|\Gamma_{\gamma^{*} \rightarrow q \bar{q}}^{(0)}\right|^{2}\right|_{\substack{\boldsymbol{r} \rightarrow \boldsymbol{r}-\boldsymbol{\ell} \\ \boldsymbol{k} \rightarrow \boldsymbol{k}+\boldsymbol{\ell}}} \frac{1}{(\boldsymbol{\ell}-\boldsymbol{r})^{4}} \frac{4 C_{A} \boldsymbol{r}^{2}(\boldsymbol{\ell}-\boldsymbol{r})^{2}}{\boldsymbol{\ell}^{2}} .
$$

Here the last factor is the BFKL kernel (without the gluon trajectory function)

$$
\mathcal{K}_{\text {real }}(\boldsymbol{r}-\boldsymbol{\ell}, \boldsymbol{r})=\frac{C_{A}}{\pi} \frac{\boldsymbol{r}^{2}(\boldsymbol{\ell}-\boldsymbol{r})^{2}}{\ell^{2}}
$$

and $1 /(\boldsymbol{\ell}-\boldsymbol{r})^{4}$ are the propagators of the (reggeized) gluons. Note that in the Born level vertex functions the transverse momenta are shifted from $\boldsymbol{r}$ to $\boldsymbol{r}-\boldsymbol{\ell}$ and from $\boldsymbol{k}$ to $\boldsymbol{k}+\boldsymbol{\ell}$. 
The color factor $C_{A}$ signals that the $C_{F}$ part of our matrix elements does not contribute to our subtraction of the central region. In the limit where the centrally emitted gluon is soft $(\boldsymbol{\ell} \ll \boldsymbol{r})$, we obtain

$$
\left.\left|\Gamma_{\gamma^{*} \rightarrow q \bar{q} g}^{(0)}\right|^{2}\right|_{\mathrm{LL}} ^{\mathrm{soft}}=\left|\Gamma_{\gamma^{*} \rightarrow q \bar{q}}^{(0)}(\alpha, \boldsymbol{k})\right|^{2} \frac{4 C_{A}}{\boldsymbol{\ell}^{2}} .
$$

The same result is obtained from the soft limit (141), if we go into the central region limit $\alpha_{\ell} \ll 1$.

\section{SUBTRACTION OF THE CENTRAL REGION}

After having calculated the various divergent limits of our matrix elements, we now turn to the subtraction of the central region. As we have discussed in section [1], contributions to the total cross section are divided according to the number of large rapidity gaps. What we have presented in the last two sections are the results for the process $\gamma^{*}+q \rightarrow(q \bar{q} g)+q$, imposing the constraint of a large rapidity gap between the $(q \bar{q} g)$ system and the quark. These results therefore contain, as a special case, still the configuration where the $q \bar{q} g$ system contains a second rapidity gap between the $q \bar{q}$ pair and the gluon: this piece has to be removed since it counts as a configuration with two gaps and, in order $\alpha_{s}^{3}$ of the total cross section, it entirely belongs to the leading-ln $s$ approximation (13).

In order to make the separation more explicit, we first divide the phase space of the produced gluon into 'upper' and 'lower' halves. Introducing

$$
\alpha_{\ell}^{c u t}=\frac{|\ell|}{\sqrt{s}},
$$

the 'upper' region $\alpha_{\ell}^{\text {cut }}<\alpha_{\ell}<1-\alpha$ consists of the 'upper' half of the central region plus the fragmentation region of the incoming photon, whereas the 'lower' region $\alpha_{\ell}<\alpha_{\ell}^{c u t}$ contains the lower half of the central region plus the fragmentation region of the quark. In the upper region we write:

$$
\begin{aligned}
& \int\left|\Gamma_{\gamma^{*} \rightarrow q \bar{q} g}^{(0)}\right|^{2} d \phi_{q \bar{q} g} \frac{s d \beta_{r}}{2 \pi} \Theta\left(\alpha_{\ell}-\alpha_{\ell}^{\mathrm{cut}}\right) \\
& =\int d \boldsymbol{k} d \boldsymbol{\ell} \int_{0}^{1} d \alpha \int_{0}^{1-\alpha} d \alpha_{\ell} \mathcal{I}_{3}\left(\alpha_{\ell}, \boldsymbol{\ell} ; \alpha, \boldsymbol{k} ; \boldsymbol{r}, Q\right) \Theta\left(\alpha_{\ell}-\alpha_{\ell}^{\mathrm{cut}}\right)
\end{aligned}
$$

Here $\mathcal{I}_{3}$ denotes the full integrand involving the sum of the matrix elements of the threeparticle final state.

The division into central region and fragmentation region is closely related to the choice of the energy scale $s_{0}$. In (13) we have introduced the energy scale $s_{0}$ : since a change in $s_{0}$ can be absorbed into the NLO impact factors $\Phi_{A}^{(1)}$ and $\Phi_{B}^{(1)}$, one might expect that there exists some freedom in choosing this scale. We adopt the simplest choice, namely a constant value which is independent of kinematic variables inside the impact factors. Examples for $s_{0}$ include the (negative) virtuality of the $t$-channel gluon, $\boldsymbol{r}^{2}$, or the (negative) mass of the photon, $Q^{2}$. 
In order to define the $q \bar{q} g$ contribution to the photon impact factor, $\Phi_{\gamma^{*}}^{(1, \text { real })}$, we have to subtract, starting from (149), the leading-ln $s$ piece with the energy scale $s_{0}$. This can be achieved by considering the central region limit (145) of the matrix element which implies

$$
\left.\mathcal{I}_{3}\left(\alpha_{\ell}, \boldsymbol{\ell} ; \alpha, \boldsymbol{k} ; \boldsymbol{r}, Q\right)\right|_{\mathrm{LL}}=\mathcal{I}_{2}(\alpha, \boldsymbol{k}+\boldsymbol{\ell} ; \boldsymbol{r}-\boldsymbol{l}, Q) \frac{1}{(2 \pi)^{2-2 \epsilon}(\boldsymbol{\ell}-\boldsymbol{r})^{4}} \mathcal{K}_{\text {real }}(\boldsymbol{r}-\boldsymbol{\ell}, \boldsymbol{r}) \frac{1}{\alpha_{\ell}} .
$$

We recognize the integrand $\mathcal{I}_{2}$ occurring in the LO impact factor $(27)$ with the value of the reggeon momentum being shifted to $\boldsymbol{r} \rightarrow \boldsymbol{r}-\boldsymbol{\ell}$ (since the momentum $\boldsymbol{\ell}$ is carried by the outgoing gluon), the propagators associated to the $t$-channel (reggeized) gluons, the measure factor $1 /(2 \pi)^{2-2 \epsilon}$ required by the definition (2), the real part of the BFKL kernel, and, finally, a factor $1 / \alpha_{\ell}$. It is this factor $1 / \alpha_{\ell}$ which, when the integration over $\alpha_{\ell}$ is done, provides the logarithm of the energy. Our aim of finding the LL piece with energy scale $s_{0}$ leads to the following $\alpha_{\ell}$ integral:

$$
\int_{0}^{\alpha_{\ell}^{\max }} \frac{d \alpha_{\ell}}{\alpha_{\ell}} \Theta\left(\alpha_{\ell}-\alpha_{\ell}^{\text {cut }}\right)=\frac{1}{2} \log \frac{\left(\alpha_{\ell}^{\max }\right)^{2} s}{\ell^{2}} .
$$

with $\alpha_{\ell}^{\max } \equiv|\ell| / \sqrt{s_{0}}$. Therefore, we define as genuine real emission contribution to the photon impact factor the expression

$$
\begin{aligned}
\Phi_{\gamma^{*}}^{(1, \text { real })}= & \int d \boldsymbol{k} d \boldsymbol{\ell} \int_{0}^{1} d \alpha \int_{\alpha_{\ell}^{\text {cut }}}^{\infty} d \alpha_{\ell}\left[\mathcal{I}_{3}\left(\alpha_{\ell}, \boldsymbol{\ell} ; \alpha, \boldsymbol{k} ; \boldsymbol{r}, Q\right) \Theta\left(1-\alpha-\alpha_{\ell}\right)\right. \\
& \left.-\mathcal{I}_{2}(\alpha, \boldsymbol{k}+\boldsymbol{\ell} ; \boldsymbol{r}-\boldsymbol{l}, Q) \frac{1}{(2 \pi)^{2-2 \epsilon}(\boldsymbol{\ell}-\boldsymbol{r})^{4}} \mathcal{K}_{\text {real }}(\boldsymbol{r}-\boldsymbol{\ell}, \boldsymbol{r}) \frac{1}{\alpha_{\ell}} \Theta\left(\frac{|\boldsymbol{\ell}|}{\sqrt{s_{0}}}-\alpha_{\ell}\right)\right] .
\end{aligned}
$$

In this definition of our impact factor, the following remark is in place. With our choice of a constant energy scale, which has led to the subtraction, we are adding (in the LL term) and subtracting (in $\Phi_{\gamma^{*}}^{(1, \text { real) }}$ ) a region of the phase space which is kinematically forbidden. In fact, the LL subtraction (last line of Eq. (152)) includes the region of phase space where $\alpha_{\ell}>1-\alpha$. It is important to make sure that this "forbidden" region $|\ell|>(1-\alpha) \sqrt{s_{0}}, 1-\alpha<\alpha_{\ell}<$ $|\ell| / \sqrt{s_{0}}$ contributes a finite term and does not introduce new divergences. The finiteness

results from the "good" UV behaviour of the LO impact factor, namely $\Phi_{\gamma^{*}}^{(0)}(\boldsymbol{r}-\boldsymbol{\ell}) \rightarrow$ const for $\ell \rightarrow \infty$ (up to logarithms). After multiplication with the propagator $\sim 1 / \ell^{4}$ and with the factor $\log \left[|\ell| /(1-\alpha) \sqrt{s_{0}}\right]$ (which is due to the $\alpha_{\ell}$ integral), the resulting $\ell$ integral is finite. This situation is quite different from the case of partonic impact factors [6,7], where $\Phi_{q, g}^{(0)}(\boldsymbol{r}-\boldsymbol{\ell}) \sim \boldsymbol{\ell}^{2}$, and the $\boldsymbol{\ell}$ integral, after including the gluon propagators and the logarithmic factor, leads to a double pole in $1 / \epsilon$.

\section{FINITE COMBINATIONS}

In the previous section we have suggested a suitable form of the LL subtraction and thus specified the real emission contribution to the impact factor. What remains are the infrared divergences which have to cancel once we combine real and virtual corrections. In this final 
section of our paper we show explicitly the cancellation of all the IR singularities, and we present expressions consisting of well defined finite terms.

Given that all the singular terms of the virtual corrections are proportional to the LO impact factor (27), as one can see from Eq. (30), it is convenient to study the singular regions of the phase space integrals of the real corrections by integrating first only over the gluon variables. In fact, the gluon integration generates singular terms which are in turn proportional to the LO impact factor. They will be combined with the singular virtual corrections after a proper identification of the remaining integration variables.

It is also convenient to separate the different colour structure contributions into a $C_{A}$ term and a $C_{F}$ term

$$
\mathcal{I}_{3} \equiv C_{A} \mathcal{I}_{3}^{C_{A}}+C_{F} \mathcal{I}_{3}^{C_{F}}
$$

because the LL subtraction is contained only in the former term, whilst the collinear singularities are present only in the latter. Soft singularities are found in both terms.

\section{A. $C_{A}$ term}

Let us consider the $C_{A}$ contribution to the real emission impact factor corrections, as specified in Eq. (152). We want to compute first the $\boldsymbol{\ell}$ and $\alpha_{\ell}$ integrals at fixed $\boldsymbol{k}$ and $\alpha$ :

$$
\begin{aligned}
\left.\frac{d \Phi_{\gamma^{*}}^{(1)}}{d \alpha d \boldsymbol{k}}\right|_{C_{A}}=\int d \boldsymbol{\ell} \int_{\alpha_{\ell}^{\text {cut }}}^{\infty} d \alpha_{\ell} & {\left[\mathcal{I}_{3}^{C_{A}}\left(\alpha_{\ell}, \boldsymbol{\ell} ; \alpha, \boldsymbol{k} ; \boldsymbol{r}, Q\right) \Theta\left(1-\alpha-\alpha_{\ell}\right)\right.} \\
& \left.\quad-\mathcal{I}_{2}(\alpha, \boldsymbol{k}+\boldsymbol{\ell} ; \boldsymbol{r}-\boldsymbol{l}, Q) \frac{2}{(2 \pi)^{3-2 \epsilon}} \frac{\boldsymbol{r}^{2}}{(\boldsymbol{r}-\boldsymbol{\ell})^{2} \boldsymbol{\ell}^{2}} \frac{1}{\alpha_{\ell}} \Theta\left(\frac{|\boldsymbol{\ell}|}{\sqrt{s_{0}}}-\alpha_{\ell}\right)\right] .
\end{aligned}
$$

This integral is divergent in the soft region $\alpha_{\ell} \rightarrow 0, \ell \rightarrow 0$ when $\epsilon=0$. We evaluate it in dimensional regularization by means of the subtraction method: we subtract from the integrand its soft approximation, in such a way that the resulting integral will be $\epsilon$-finite. Subsequently we re-add what we have subtracted, and we integrate this term analytically. Of course, the soft limit is not uniquely defined outside the soft region. We choose the soft approximation according to Eqs. (141, 147, 144); we extend the soft subtraction up to the kinematic limit of the gluon longitudinal momentum, i.e., $\alpha_{\ell}<1-\alpha$ :

$$
\begin{aligned}
\left.\frac{d \Phi_{\gamma^{*}}^{(1)}}{d \alpha d \boldsymbol{k}}\right|_{C_{A}} ^{\mathrm{soft}}= & \mathcal{I}_{2}(\alpha, \boldsymbol{k} ; \boldsymbol{r}, Q) \frac{2}{(2 \pi)^{3-2 \epsilon}} \\
& \times \int d \boldsymbol{\ell} \int_{\alpha_{\ell}^{\text {cut }}}^{\infty} \frac{d \alpha_{\ell}}{\alpha_{\ell}}\left[\frac{\left(\boldsymbol{\ell}-\alpha_{\ell} \boldsymbol{a}\right) \cdot\left(\boldsymbol{\ell}+\alpha_{\ell} \boldsymbol{b}\right)}{\left(\boldsymbol{\ell}-\alpha_{\ell} \boldsymbol{a}\right)^{2}\left(\boldsymbol{\ell}+\alpha_{\ell} \boldsymbol{b}\right)^{2}}-\frac{1}{\boldsymbol{\ell}^{2}} \Theta\left(\frac{|\boldsymbol{\ell}|}{\sqrt{s_{0}}}-\alpha_{\ell}\right)\right] \Theta\left(1-\alpha-\alpha_{\ell}\right) .
\end{aligned}
$$


Accordingly, we define the finite $C_{A}$ part of the real corrections to the impact factor as

$$
\begin{aligned}
& \left.\Phi_{\gamma^{*}}^{(1, \text { real })}\right|_{C_{A}} ^{\text {finite }} \equiv \int d \boldsymbol{k} d \boldsymbol{\ell} \int_{0}^{1} d \alpha \int_{0}^{\infty} d \alpha_{\ell}\left\{\left[\mathcal{I}_{3}^{C_{A}}\left(\alpha_{\ell}, \boldsymbol{\ell} ; \alpha, \boldsymbol{k} ; \boldsymbol{r}, Q\right) \Theta\left(1-\alpha-\alpha_{\ell}\right)\right.\right. \\
& \left.-\mathcal{I}_{2}(\alpha, \boldsymbol{k}+\boldsymbol{\ell} ; \boldsymbol{r}-\boldsymbol{l}, Q) \frac{2}{(2 \pi)^{3-2 \epsilon}} \frac{\boldsymbol{r}^{2}}{(\boldsymbol{r}-\boldsymbol{\ell})^{2} \boldsymbol{\ell}^{2}} \frac{1}{\alpha_{\ell}} \Theta\left(\frac{|\boldsymbol{\ell}|}{\sqrt{s_{0}}}-\alpha_{\ell}\right)\right] \\
& \left.-\mathcal{I}_{2}(\alpha, \boldsymbol{k} ; \boldsymbol{r}, Q) \frac{2}{(2 \pi)^{3-2 \epsilon}} \frac{1}{\alpha_{\ell}}\left[\frac{\left(\boldsymbol{\ell}-\alpha_{\ell} \boldsymbol{a}\right) \cdot\left(\boldsymbol{\ell}+\alpha_{\ell} \boldsymbol{b}\right)}{\left(\boldsymbol{\ell}-\alpha_{\ell} \boldsymbol{a}\right)^{2}\left(\boldsymbol{\ell}+\alpha_{\ell} \boldsymbol{b}\right)^{2}}-\frac{1}{\boldsymbol{\ell}^{2}} \Theta\left(\frac{|\boldsymbol{\ell}|}{\sqrt{s_{0}}}-\alpha_{\ell}\right)\right] \Theta\left(1-\alpha-\alpha_{\ell}\right)\right\} .
\end{aligned}
$$

Note that we have shifted to zero the lower limit of integration of $\alpha_{\ell}$ because, thanks to the LL subtraction, there is no contribution to the integral coming from the central region. Note also that the singularities of the integrand due to the denominators $\boldsymbol{\ell}-\alpha_{\ell} \boldsymbol{a}$ and $\boldsymbol{\ell}+\alpha_{\ell} \boldsymbol{b}$ are integrable, because of the scalar product in the numerator. This reflects the fact that the $C_{A}$ part is free of collinear singularities.

The expression written in Eq. (155) can be computed analytically. Since the lower limit of integration in $\alpha_{\ell}$ depends on the energy $s$, one gets an $s$ dependent result. However, because of the LL-soft subtraction to the soft term, the central region contribution is suppressed, and we expect a finite expression in the $s \rightarrow \infty$ limit. We can compute the integrals of the two terms in Eq. (155) in this limit $\left(\alpha_{\ell}^{\text {cut }} \rightarrow 0\right)$ separately because dimensional regularization promotes the divergent $\log s$ contributions into UV $\epsilon$-poles, which will cancel in the difference. We have:

$$
J_{1} \equiv \int d \boldsymbol{\ell} \int_{0}^{1-\alpha} \frac{d \alpha_{\ell}}{\alpha_{\ell}} \frac{\left(\boldsymbol{\ell}-\alpha_{\ell} \boldsymbol{a}\right) \cdot\left(\boldsymbol{\ell}+\alpha_{\ell} \boldsymbol{b}\right)}{\left(\boldsymbol{\ell}-\alpha_{\ell} \boldsymbol{a}\right)^{2}\left(\boldsymbol{\ell}+\alpha_{\ell} \boldsymbol{b}\right)^{2}}=-c_{\Gamma} \pi^{1-\epsilon} \frac{(1-\alpha)^{-2 \epsilon}}{2 \epsilon^{2}}\left((\boldsymbol{a}+\boldsymbol{b})^{2}\right)^{-\epsilon}
$$

and

$$
J_{2} \equiv \int d \boldsymbol{\ell} \int_{0}^{1-\alpha} \frac{d \alpha_{\ell}}{\alpha_{\ell}} \frac{1}{\ell^{2}} \Theta\left(\frac{|\ell|}{\sqrt{s_{0}}}-\alpha_{\ell}\right)=-\frac{\pi^{1-\epsilon}}{\Gamma(1-\epsilon)} \frac{(1-\alpha)^{-2 \epsilon}}{2 \epsilon^{2}} s_{0}^{-\epsilon} .
$$

These two integrals have to be multiplied by $\mathcal{I}_{2}$ and integrated over $\alpha$ and $\boldsymbol{k}$. After Eq. (27) we have noticed that $\mathcal{I}_{2}$ is symmetric under the change of variables $\alpha \leftrightarrow 1-\alpha, \boldsymbol{k} \leftrightarrow-\boldsymbol{\ell}-\boldsymbol{k}$, corresponding to the $q \leftrightarrow \bar{q}$ exchange; the term $(\boldsymbol{a}+\boldsymbol{b})^{2}=M^{2} / \alpha(1-\alpha)$ is also invariant under this exchange. Therefore, on the right-hand-side of Eqs. (157) and (158), we can replace $(1-\alpha)^{-2 \epsilon} \rightarrow \frac{1}{2} \alpha^{-2 \epsilon}+\frac{1}{2}(1-\alpha)^{-2 \epsilon}$, which casts the expressions into explicitly symmetric forms w.r.t. $\alpha \leftrightarrow 1-\alpha$. This manipulation will be useful when combining real and virtual corrections.

In conclusion, the divergent $C_{A}$ part of the real corrections to the impact factor is

$$
\begin{aligned}
\left.\Phi_{\gamma^{*}}^{(1, \text { real })}\right|_{C_{A}} ^{\text {divergent }} \equiv & \int d \boldsymbol{k} \int_{0}^{1} d \alpha \mathcal{I}_{2}(\alpha, \boldsymbol{k} ; \boldsymbol{r}, Q) \frac{2}{(2 \pi)^{3-2 \epsilon}}\left(J_{1}-J_{2}\right) \\
= & \int d \boldsymbol{k} \int_{0}^{1} d \alpha \mathcal{I}_{2}(\alpha, \boldsymbol{k} ; \boldsymbol{r}, Q) \frac{c_{\Gamma}}{(4 \pi)^{2-\epsilon}}\left\{\frac{2}{\epsilon}\left[\log M^{2}-\log s_{0}-\log \alpha(1-\alpha)\right]\right. \\
& \left.+\left[\left(\log s_{0}+\log \alpha(1-\alpha)\right)^{2}-\log ^{2} M^{2}\right]+\mathcal{O}(\epsilon)\right\} .
\end{aligned}
$$

For consistency, the variables $\alpha$ and $\boldsymbol{k}$ of the above equation have to be identified with the analogous variables of Eq. (37). 


\section{B. $C_{F}$ term}

Let us now consider the $C_{F}$ contribution to the real emission impact factor corrections. The first thing to note is that, since the BFKL kernel is proportional to $C_{A}$, the LL subtraction of Eq. (152) does not affect the $C_{F}$ part. Therefore, the whole $C_{F}$ part of $\mathcal{I}_{3}$ contributes to the impact factor. In addition, we can shift the lower limit $\alpha_{\ell}^{\text {cut }}$ for the $\alpha_{\ell}$ integral to zero, just because the central region does not contribute in this case.

The $C_{F}$ term contains both soft and collinear divergences. We separate the singular contributions by means of the subtraction method. In what follows, the last two parameters of $\mathcal{I}_{2}$ are always $\boldsymbol{r}, Q$. Therefore we simplify the notation by indicating $\mathcal{I}_{2}(\alpha, \boldsymbol{k}) \equiv \mathcal{I}_{2}(\alpha, \boldsymbol{k} ; \boldsymbol{r}, Q)$.

We start by identifying the soft singular term. This is done by subtracting from $\mathcal{I}_{3}^{C_{F}}$ its soft limit and then re-adding it again. We remark again that, beyond the soft region, the limit of integration is not uniquely defined. In practice, by using the expression (142), we adopt an asymmetric function with respect to the $q \leftrightarrow \bar{q}$ exchange at fixed non-vanishing gluon momentum:

$$
\begin{aligned}
\left.\frac{d \Phi_{\gamma^{*}}^{(1)}}{d \alpha d \boldsymbol{k}}\right|_{C_{F}} ^{\text {soft }} & =\mathcal{I}_{2}(\alpha, \boldsymbol{k}) \frac{2(\boldsymbol{a}+\boldsymbol{b})^{2}}{(2 \pi)^{3-2 \epsilon}} \int d \boldsymbol{\ell} \int_{0}^{1-\alpha} d \alpha_{\ell} \frac{\alpha_{\ell}}{\left(\boldsymbol{\ell}-\alpha_{\ell} \boldsymbol{a}\right)^{2}\left(\boldsymbol{\ell}+\alpha_{\ell} \boldsymbol{b}\right)^{2}} \\
& =\mathcal{I}_{2}(\alpha, \boldsymbol{k}) \frac{c_{\Gamma}}{(4 \pi)^{2-\epsilon}} \frac{2}{\epsilon^{2}}\left[\alpha^{-2 \epsilon}+(1-\alpha)^{-2 \epsilon}\right]\left[\frac{M^{2}}{\alpha(1-\alpha)}\right]^{-\epsilon}
\end{aligned}
$$

where in the last equality a symmetrization in $\alpha$, as indicated after Eq. (158), has been performed.

Both the full $C_{F}$ term $\propto \mathcal{I}_{3}^{C_{F}}$ and its soft limit (160) have a divergent behaviour when the gluon is either collinear to the outgoing quark or antiquark. Also for the evaluation of the collinear divergences we adopt the subtraction method. For the case of the gluon being collinear to the antiquark $(g \| \bar{q})$, we derive the limiting expression of the original integrand (i.e., without soft subtraction yet) from Eq. (137), which yields $\left(\boldsymbol{\ell}^{\prime}=\boldsymbol{\ell}+\alpha_{\ell} \boldsymbol{b}\right)$

$$
\begin{aligned}
\left.\frac{d \Phi_{\gamma^{*}}^{(1)}}{d \alpha d \boldsymbol{k}}\right|_{C_{F}} ^{\mathrm{coll}, \bar{q}} & =\mathcal{I}_{2}(\alpha, \boldsymbol{k}) \int \frac{d \boldsymbol{\ell}}{(2 \pi)^{3-2 \epsilon}} \int_{0}^{1-\alpha} \frac{d \alpha_{\ell}}{(1-\alpha)} P_{g q}\left(\frac{\alpha_{\ell}}{1-\alpha}, \epsilon\right) \frac{\Theta\left(\alpha_{\ell} \Lambda-\left|\ell^{\prime}\right|\right)}{\ell^{\prime 2}} \\
& =\mathcal{I}_{2}(\alpha, \boldsymbol{k}) \frac{\alpha^{-2 \epsilon}+(1-\alpha)^{-2 \epsilon}}{(4 \pi)^{2-\epsilon} \Gamma(1-\epsilon)} \frac{\Lambda^{-2 \epsilon}}{\epsilon}\left[\frac{1}{\epsilon}+\frac{2}{1-2 \epsilon}-\frac{1}{2}\right],
\end{aligned}
$$

where we have decided to perform the collinear subtraction only in a cone - specified by the $\Theta$ function — whose axis coincides with the gluon momentum being parallel to the antiquark momentum, and the vertex sits on the soft point. The "opening angle" of the cone is parametrized by a cutoff $\Lambda$ : the bigger $\Lambda$, the wider the domain of the collinear subtraction.

In order to end up with a finite integral, we have to subtract also the collinear singularities 
of the soft subtraction term (160). For the $g \| \bar{q}$ we have (see Eq. (139):

$$
\begin{aligned}
\left.\frac{d \Phi_{\gamma^{*}}^{(1)}}{d \alpha d \boldsymbol{k}}\right|_{C_{F}} ^{\text {soft,coll, } \bar{q}} & =\mathcal{I}_{2}(\alpha, \boldsymbol{k}) \frac{2}{(2 \pi)^{3-2 \epsilon}} \int_{0}^{1-\alpha} \frac{d \alpha_{\ell}}{\alpha_{\ell}} \int d \boldsymbol{\ell} \frac{\Theta\left(\alpha_{\ell} \Lambda-\left|\ell^{\prime}\right|\right)}{\boldsymbol{\ell}^{\prime 2}} \\
& =\mathcal{I}_{2}(\alpha, \boldsymbol{k}) \frac{1}{(4 \pi)^{2-\epsilon} \Gamma(1-\epsilon)}\left[\alpha^{-2 \epsilon}+(1-\alpha)^{-2 \epsilon}\right] \frac{\Lambda^{-2 \epsilon}}{\epsilon^{2}}
\end{aligned}
$$

The soft-collinear subtraction uses the same cone as defined for the collinear one. It is important that Eq. (162) can be obtained both as a collinear limit of Eq. (160) and as a soft limit of Eq. (161). This guarantees not only that the collinear singularities introduced by the soft subtraction are subtracted, but also that the soft singularities introduced by the collinear subtraction are properly removed.

Note that the collinear term (161) is proportional to $\mathcal{I}_{2}(\alpha, \boldsymbol{k})$. This is a consequence of the convenient choice of variable employed to parametrize the outgoing particles. In fact, when the gluon is collinear to the antiquark, the $g \bar{q}$ system has to be treated as a single entity carrying the sum of quantum numbers and momenta of the constituting particles. In this case, the $g \bar{q}$ longitudinal momentum fraction and transverse momentum are $1-\alpha$ and $\boldsymbol{-} \boldsymbol{k}$ respectively. The integration over the gluon variables can therefore be independently performed leaving the LO integrand $\mathcal{I}_{2}$ factored out.

The treatment of the $g \| q$ collinear singularity can be performed in the same way as for the $g \| \bar{q}$ collinear case. There are only two small differences: first of all, the collinear limit of the integrand $\mathcal{I}_{3}$ is proportional to $\mathcal{I}_{2}\left(\alpha+\alpha_{\ell}, \boldsymbol{k}+\boldsymbol{\ell}\right)$, which involves explicitly the gluon variables $\alpha_{\ell}$ and $\boldsymbol{\ell}$. This is due to our set of variables that does not describe the $q g$ system and the single gluon independently. A more suitable set of variable in this case is simply obtained by changing the $q \leftrightarrow \bar{q}$ labels:

$$
\begin{aligned}
\bar{\alpha} & \equiv 1-\alpha-\alpha_{\ell} \\
\overline{\boldsymbol{k}} & \equiv-\boldsymbol{r}-\boldsymbol{k}-\boldsymbol{\ell} \\
\boldsymbol{\ell}^{\prime \prime} & \equiv \boldsymbol{\ell}-\alpha_{\ell} \boldsymbol{a} .
\end{aligned}
$$

From Eq. (161) one gets

$$
\begin{aligned}
\left.\frac{d \Phi_{\gamma^{*}}^{(1)}}{d \bar{\alpha} d \overline{\boldsymbol{k}}}\right|_{C_{F}} ^{\mathrm{coll}, q}= & \mathcal{I}_{2}(\bar{\alpha}, \overline{\boldsymbol{k}}) \int \frac{d \boldsymbol{\ell}}{(2 \pi)^{3-2 \epsilon}} \int_{0}^{1-\bar{\alpha}} \frac{d \alpha_{\ell}}{(1-\bar{\alpha})} P_{g q}\left(\frac{\alpha_{\ell}}{1-\bar{\alpha}}, \epsilon\right) \\
& \times\left(\frac{1-\bar{\alpha}}{1-\bar{\alpha}-\alpha_{\ell}}\right)^{2} \frac{\Theta\left(\alpha_{\ell} \Lambda-\frac{1-\bar{\alpha}-\alpha_{\ell}}{1-\bar{\alpha}}\left|\ell^{\prime \prime}\right|\right)}{\ell^{\prime \prime 2}} \\
= & \mathcal{I}_{2}(1-\bar{\alpha},-\boldsymbol{r}-\overline{\boldsymbol{k}}) \frac{\bar{\alpha}^{-2 \epsilon}+(1-\bar{\alpha})^{-2 \epsilon}}{(4 \pi)^{2-\epsilon} \Gamma(1-\epsilon)} \frac{\Lambda^{-2 \epsilon}}{\epsilon}\left[\frac{1}{\epsilon}+\frac{2}{1-2 \epsilon}-\frac{1}{2}\right] .
\end{aligned}
$$

The additional factors in the second line on the rhs of Eq. (164) (when compared to the corresponding line of Eq. (161)) are due to the fact that

$$
\boldsymbol{\ell}+\left.\alpha_{\ell} \boldsymbol{b}\right|_{\substack{\alpha \rightarrow \bar{\alpha} \\ \boldsymbol{k} \rightarrow \overline{\boldsymbol{k}}}}=\frac{1-\bar{\alpha}-\alpha_{\ell}}{1-\bar{\alpha}}\left(\boldsymbol{\ell}-\alpha_{\ell} \boldsymbol{a}\right) .
$$


The second difference is that, considering the soft-collinear subtraction, we cannot use the soft limit of Eq. (164) at fixed $\bar{\alpha}$ and $\overline{\boldsymbol{k}}$, because this does not correspond to the collinear limit of the soft approximation (160). The right way of subtracting the soft singularity of (164) and at the same time the $g \| q$ collinear singularity of (160) is to take the collinear limit of the latter:

$$
\begin{aligned}
\left.\frac{d \Phi_{\gamma^{*}}^{(1)}}{d \alpha d \boldsymbol{k}}\right|_{C_{F}} ^{\text {soft,coll }, q} & =\mathcal{I}_{2}(\alpha, \boldsymbol{k}) \frac{2}{(2 \pi)^{3-2 \epsilon}} \int_{0}^{1-\alpha} \frac{d \alpha_{\ell}}{\alpha_{\ell}} \int d \boldsymbol{\ell} \frac{\Theta\left(\alpha_{\ell} \Lambda-\left|\ell^{\prime \prime}\right|\right)}{\ell^{\prime 2^{2}}} \\
& =\mathcal{I}_{2}(\alpha, \boldsymbol{k}) \frac{1}{(4 \pi)^{2-\epsilon} \Gamma(1-\epsilon)}\left[\alpha^{-2 \epsilon}+(1-\alpha)^{-2 \epsilon}\right] \frac{\Lambda^{-2 \epsilon}}{\epsilon^{2}}
\end{aligned}
$$

In conclusion, we write the finite part of the $C_{F}$ term of the impact factor by subtracting, from $\mathcal{I}_{3}^{C_{F}}$, the unintegrated expressions of Eqs. (160, 161, 162, 164, 166):

$$
\begin{aligned}
& \left.\Phi_{\gamma^{*}}^{(1, \text { real })}\right|_{C_{F}} ^{\text {finite }} \equiv \int d \boldsymbol{k} d \boldsymbol{\ell} \int_{0}^{1} d \alpha \int_{0}^{1-\alpha} d \alpha_{\ell}\left\{\mathcal{I}_{3}^{C_{F}}\left(\alpha_{\ell}, \boldsymbol{\ell} ; \alpha, \boldsymbol{k} ; \boldsymbol{r}, Q\right)\right. \\
& -\mathcal{I}_{2}(\alpha, \boldsymbol{k}) \frac{2(\boldsymbol{a}+\boldsymbol{b})^{2}}{(2 \pi)^{3-2 \epsilon}} \frac{\alpha_{\ell}}{\left(\boldsymbol{\ell}-\alpha_{\ell} \boldsymbol{a}\right)^{2}\left(\boldsymbol{\ell}+\alpha_{\ell} \boldsymbol{b}\right)^{2}} \\
& -\mathcal{I}_{2}(\alpha, \boldsymbol{k}) \frac{1}{(2 \pi)^{3-2 \epsilon}}\left[\frac{1}{1-\alpha} P_{g q}\left(\frac{\alpha_{\ell}}{1-\alpha}, \epsilon\right)-\frac{2}{\alpha_{\ell}}\right] \frac{\Theta\left(\alpha_{\ell} \Lambda-\left|\boldsymbol{\ell}+\alpha_{\ell} \boldsymbol{b}\right|\right)}{\left(\boldsymbol{\ell}+\alpha_{\ell} \boldsymbol{b}\right)^{2}} \\
& -\left[\mathcal{I}_{2}\left(\alpha+\alpha_{\ell}, \boldsymbol{k}+\boldsymbol{\ell}\right) \frac{\alpha+\alpha_{\ell}}{\alpha^{2}} P_{g q}\left(\frac{\alpha_{\ell}}{\alpha+\alpha_{\ell}}, \epsilon\right) \Theta\left(\alpha_{\ell} \Lambda-\frac{\alpha}{\alpha+\alpha_{\ell}}\left|\boldsymbol{\ell}-\alpha_{\ell} \boldsymbol{a}\right|\right)\right. \\
& \left.\quad-\mathcal{I}_{2}(\alpha, \boldsymbol{k}) \frac{2}{\alpha_{\ell}} \Theta\left(\alpha_{\ell} \Lambda-\left|\boldsymbol{\ell}-\alpha_{\ell} \boldsymbol{a}\right|\right)\right] \frac{1}{(2 \pi)^{3-2 \epsilon}\left(\boldsymbol{\ell}-\alpha_{\ell} \boldsymbol{a}\right)^{2}} .
\end{aligned}
$$

The divergent part of the $C_{F}$ term is obtained by re-adding the subtracted pieces in their integrated form. In doing this, we identify the $1-\bar{\alpha}$ and $-\boldsymbol{r}-\overline{\boldsymbol{k}}$ variables of Eq. (164) with the variables $\alpha$ and $\boldsymbol{k}$ of Eqs. (161, 162, 166) (and later with $\alpha$ and $\boldsymbol{k}$ of Eq. (37)), so that

$$
\begin{aligned}
\left.\Phi_{\gamma^{*}}^{(1, \text { real })}\right|_{C_{F}} ^{\text {divergent }}= & \int d \boldsymbol{k} \int_{0}^{1} d \alpha \mathcal{I}_{2}(\alpha, \boldsymbol{k}) \frac{c_{\Gamma}}{(4 \pi)^{2-\epsilon}}\left\{\frac{4}{\epsilon^{2}}+\frac{1}{\epsilon}\left[6-4 \log M^{2}\right]\right. \\
& \left.+2\left[8+\log ^{2} M^{2}-3 \log \Lambda^{2}-3 \log \alpha(1-\alpha)+\log ^{2} \frac{\alpha}{1-\alpha}\right]+\mathcal{O}(\epsilon)\right\} .
\end{aligned}
$$

The final expression for the one-loop correction to the photon impact factor can be obtained by summing the real contributions given in Eqs. (156, 159, 167, 168) to the virtual corrections $\Phi_{\gamma^{*}}^{(1, \text { virtual })}$ of [1]. One can check the cancellation of soft and collinear singularities by combining the singular piece of the virtual corrections (37) with the $\epsilon$-poles of Eqs. (159) and (168). The cancellation of double and single pole is straightforward.

In conclusion, the final expression of the impact factor at NLO can be obtained by summing the finite part of the virtual corrections, the scale dependent term (44) and the extra term (41) as discussed in section III, the finite terms of Eqs. (159) and (168), and the 
integrals in Eqs. (156) and (167) (evaluated at $\epsilon=0)$ :

$$
\begin{aligned}
\Phi_{\gamma^{*}}^{(1)}= & \left.\Phi_{\gamma^{*}}^{(1, \text { virtual })}\right|^{\text {finite }}-\frac{2 \Phi_{\gamma^{*}}^{(0)}}{(4 \pi)^{2}}\left\{\beta_{0} \ln \frac{\boldsymbol{r}^{2}}{\mu^{2}}+C_{F} \ln \left(\boldsymbol{r}^{2}\right)\right\} \\
& +\frac{1}{(4 \pi)^{2}} \int d \boldsymbol{k} \int_{0}^{1} d \alpha \mathcal{I}_{2}(\alpha, \boldsymbol{k})\left\{C_{A}\left[\ln ^{2} \alpha(1-\alpha) s_{0}-\ln ^{2} M^{2}\right]\right. \\
& \left.+2 C_{F}\left[8-3 \ln \alpha(1-\alpha) \Lambda^{2}+\ln ^{2} M^{2}+\ln ^{2} \frac{\alpha}{1-\alpha}\right]\right\} \\
& +\left.C_{A} \Phi_{\gamma^{*}}^{(1, \text { real })}\right|_{C_{A}} ^{\text {finite }}+\left.C_{F} \Phi_{\gamma^{*}}^{(1, \text { real })}\right|_{C_{F}} ^{\text {finite }} .
\end{aligned}
$$

\section{CONCLUSIONS}

In this third part of our program of calculating the NLO corrections to the photon impact factor we first have presented a list of the real corrections, the $q \bar{q} g$ intermediate state inside the impact factor. This completes the results of the paper [2] which contained the longitudinal photon only. We then have identified and computed the divergent parts of the phase space integral of the real corrections: in addition to the infrared singularities which are due to collinear and soft configurations of the produced gluon there is a logarithmic divergence related to the $\ln s$-piece of the central region. Removal of the latter part of the real corrections introduces the energy scale $s_{0}$ which represents a central element of the NLO corrections. Finally, we have combined the real corrections with the divergent parts of the virtual corrections: these infrared finite combinations are the main results of this paper.

With these calculations we have essentially completed the analytic part of our program. What remains are numerical steps: the phase space integrals both for the $q \bar{q}$ state (which includes the finite pieces of the virtual corrections) and the $q \bar{q} g$ state. Certain parts of these integrals can be done analytically, but, as a general strategy, we define standard integrals and express our integrands in terms of these expressions. The final evaluations have to be done with the computer.

Once this is solved technically, the photon impact factor allows us to compute the total cross section for $\gamma^{*} \gamma^{*}$-scattering to next-to-leading logarithmic accuracy in $s$. This will be the first consistent calculation of a cross section to this order since the NLO corrections to the BFKL kernel were first published [8,9]. Taking advantage of the recently obtained impact factor for forward jets [10] in the same limit we may apply the photon impact factor also to the calculation of the forward jet cross section at HERA.

We have emphasized already before, that interest in the NLO calculation of the photon impact factor comes from various directions. A prominent example is the photon wave function picture. In our analysis [2] of the real corrections we have extracted the new $q \bar{q} g$ Fock component of the photon wave function which, most conveniently, is expressed in configuration space. In the present paper we have studied the cancellation of divergences, but this was done on momentum space. It will therefore be necessary to translate these calculations into configuration space. Furthermore, in order to obtain a clearer geometrical picture of how color charge is distributed inside the virtual photon, we need to take a closer look into the various pieces found in [2] and in this paper. Another question of interest 
of the photon wave function picture is the form of the NLO correction to the $q \bar{q}$ Fock component and of the $q \bar{q}$ dipole cross section: do they destroy some of the main features of the LO calculations, for example the conservation of the transverse dipole size during the interaction with the target? Answers to these questions are to be found in our virtual corrections, but they require further theoretical efforts.

\section{ACKNOWLEDGMENTS}

Helpful conversations with C.-F. Qiao are gratefully acknowledged. S.G. wishes to thank the II. Institut für Theoretische Physik of the Universität Hamburg where large parts of this work were done for kind hospitality.

[1] J. Bartels, S. Gieseke and C. F. Qiao, Phys. Rev. D 63 (2001) 056014 [Erratum-ibid. D 65 (2001) 079902] hep-ph/0009102].

[2] J. Bartels, S. Gieseke and A. Kyrieleis, Phys. Rev. D 65 (2002) 014006 hep-ph/0107152.

[3] E. A. Kuraev, L. N. Lipatov and V. S. Fadin, Sov. Phys. JETP 45 (1977) 199 [Zh. Eksp. Teor. Fiz. 72 (1977) 377]; I. I. Balitsky and L. N. Lipatov, Sov. J. Nucl. Phys. 28 (1978) 822 [Yad. Fiz. 28 (1978) 1597].

[4] V. S. Fadin and A. D. Martin, Phys. Rev. D 60 (1999) 114008 hep-ph/9904505.

[5] S. Catani and M. H. Seymour, Nucl. Phys. B 485 (1997) 291 [Erratum-ibid. B 510 (1997) 503] hep-ph/9605323.

[6] M. Ciafaloni, Phys. Lett. B 429 (1998) 363 hep-ph/9801322; M. Ciafaloni and D. Colferai, Nucl. Phys. B 538 (1999) 187 hep-ph/9806350.

[7] V. S. Fadin, R. Fiore, M. I. Kotsky and A. Papa, Phys. Rev. D 61 (2000) 094005 [hepph/9908264; Phys. Rev. D 61 (2000) 094006 hep-ph/9908265].

[8] V. S. Fadin and L. N. Lipatov, Phys. Lett. B 429 (1998) 127 hep-ph/9802290.

[9] G. Camici and M. Ciafaloni, Phys. Lett. B 412 (1997) 396 [Erratum-ibid. B 417 (1998) 390] hep-ph/9707390.

[10] J. Bartels, D. Colferai and G. P. Vacca, Eur. Phys. J. C 24 (2002) 83 hep-ph/0112283; hep-ph/0206290. 